\title{
Quasi-varieties and Initial Semantics for Hybridized Institutions
}

\author{
Răzvan Diaconescu \\ Simion Stoilow Institute of Mathematics of the Romanian Academy
}

\begin{abstract}
We define and develop the concept of quasi-variety for models of hybrid logics and we apply this for determining initial semantics for classes of hybrid logics theories. The hybrid logic is considered here in a very general sense, internal to abstract institutions (in the sense of the so-called institution theory of Goguen and Burstall). This means our result is applicable to a wide variety of hybrid logics including for example those resulting from the various kinds of combinations between conventional hybrid logics and various other logical systems.
\end{abstract}

\section{Introduction}

Hybrid logics [7] are a brand of modal logics that provides appropriate syntax for the possible worlds semantics in a simple and very natural way through the so-called nominals. This has several advantages from the point of view of logic and formal specification. For example it has been argued [10] that hybrid logics allow a more uniform proof theory than non-hybrid modal logics. Also in specifications of dynamic systems the possibility of explicit reference to specific states of the model is a very necessary feature.

Historically, hybrid logic was introduced in [32] and further developed in works such as $[4,10,31]$ etc. Moreover recently hybrid logic has been developed [30] at an abstract institution theoretic level. Institution theory [20] is a categorical abstract model theory that arose about three decades ago within specification theory as a response to the explosion in the population of logics in use there, its original aim being to develop as much computing science as possible in a general uniform way independently of particular logical systems. This has now been achieved to an extent even greater than originally thought, as institution theory became the most fundamental mathematical theory underlying algebraic specification theory (in its wider meaning), also being increasingly used in other areas of computer science. Moreover, institution theory constitutes a major trend in the so-called 'universal logic' (in the sense envisaged by Jean-Yves Béziau [6]) which is considered by many a true renaissance of mathematical logic.

The development in [30], which extends the previous work on institution-independent possible worlds semantics of [19] to nominals and multi-modalities, abstracts away the details, both at the syntactic and the semantic levels, that are independent of the very essence of the hybrid logic idea. This has several benefits. One is a general benefit of institution theoretic developments, namely that the theoretical development is not hindered by logical details that are often irrelevant. Another benefit is the applicability of the results to a wide variety of concrete instances, many of which could be regarded as combinations between concrete versions of hybrid logics with other logical systems. Such combinations meet the influential Goguen thesis

Email address: Razvan.Diaconescu@imar.ro (Răzvan Diaconescu) 
(see [21]): the combinations of computing paradigms are based upon the combinations of the underlying logical paradigms.

Initial semantics $[23,25]$ is one of the oldest, most notorious, and most developed formal specification methodologies. In its conventional forms it directly supports execution through rewriting, thus achieving a high level of integration between the specification and the formal verification levels. The initial semantics methodology has spread much beyond its original context, that of traditional equational specification, to a variety of modern more sophisticated logical contexts. Moreover initial semantics plays the foundational role in logic programming (there known as 'least Herbrand models') [27]. One of the main methods to establish initial semantics is that of the so-called 'quasi-varieties' (i.e. classes of models closed under 'submodels' and products) that were first systematically studied in [28]; in their algebraic version they also play an important role in general or universal algebra [24]. General institution theoretic approaches to initial semantics and quasi-varieties that are computing science motivated may be found in $[14,35]$ and they are related to general approaches to quasi-varieties from categorical model theory (e.g. [3]).

The generality of our approach to hybrid logics requires the general approach to quasi-varieties mentioned above. While in general the concept of product of models is fairly straightforward, the concept of 'sub-model' needs much more consideration. As the reader will see by herself in this paper, this is very much the case for hybrid logics where establishing an appropriate concept of sub-model is the most difficult part of the whole enterprise to define a concept of quasi-variety for hybrid logics models. In $[3,35]$ the concept of sub-model is usually handled through the categorical concept of 'factorisation system', while works such as $[14,33]$ prefer the so-called 'inclusion systems' of [18] which are a variant of the former that essentially requires a uniqueness property for the respective factorizations. Here we also prefer the latter.

\section{Contributions and structure of the paper.}

The paper is structured as follows:

1. The first preliminary section of the paper is devoted to the brief presentation of general institution theoretic notions that are needed by our work.

2. The second preliminary section recalls the process of 'hybridization' of institutions of [30], upgrades it with the concept of constrained models, and develops proofs that have been omitted in [30]. In brief, this means that given a 'base' institution $\mathcal{I}$, considered abstractly, we build a class of 'hybrid' institutions on top of the syntax, the semantics and the satisfaction relation of $\mathcal{I}$ by adding the features that constitute the essence of hybrid logics. The end result is a class of institutions.

3. The third technical section is devoted to preservation results along homomorphisms of models in 'hybridized' institutions and it has a rather technical scope.

4. The fourth technical section introduces the concept of sub-model for hybridized institutions by developing inclusion systems for models of hybridized institutions on top of abstract inclusion systems for the models of the base institution. This contains the most technically difficult part of our work. Within this context it also develops results on preservation by sub-models.

5. The fifth technical section develops the construction of products of models in hybridized institutions on top of (presumed) products of models of the base institution. Its second part is devoted to results on preservation by products in hybridized institutions.

6. The last technical section applies the abstract quasi-variety theorem of [14] through the results of the previous sections in order to derive a general result on existence of initial semantics for hybridized institutions. 


\section{Institutions}

Institution theory is a categorical abstract model theory, hence it is heavily based upon category theory, though the level of category theory involved is rather elementary. We assume the reader is familiar with basic notions and standard notations from elementary category theory; e.g., see [26] for an introduction to this subject. Here we recall very briefly some of them. By way of notation, $|\mathbb{C}|$ denotes the class of objects of a category $\mathbb{C}, \mathbb{C}(A, B)$ the set of arrows with domain $A$ and codomain $B$, and composition is denoted by ";" and in diagrammatic order. The category of sets (as objects) and functions (as arrows) is denoted by Set, and CAT is the category of all categories. ${ }^{1}$ The opposite of a category $\mathbb{C}$ (obtained by reversing the arrows of $\mathbb{C}$ ) is denoted $\mathbb{C}^{\mathrm{op}}$. An initial object $0_{\mathbb{C}}$ in $\mathbb{C}$ is an object such that for each other object $A \in|\mathbb{C}|$ there exist an unique arrow $0_{\mathbb{C}} \rightarrow A$. A (direct) product of a family $\left\{A_{i} \mid i \in I\right\}$ of objects in $\mathbb{C}$ consist of a family of arrows, called projections $\left\{p_{i}: A \rightarrow A_{i} \mid i \in I\right\}$ such that for any family of arrows $\left\{f_{i}: B \rightarrow A_{i} \mid i \in I\right\}$ there exists an unique arrow $f: B \rightarrow A$ such that for each $i \in I, f ; p_{i}=f_{i}$.

Institutions have been defined by Goguen and Burstall in [11], the seminal paper [20] being printed after a delay of many years. Below we recall the concept of institution which formalises the intuitive notion of logical system, including syntax, semantics, and the satisfaction between them.

Definition 2.1 (Institution). An institution $\left(\operatorname{Sign}^{\mathcal{I}}, \operatorname{Sen}^{\mathcal{I}}, \operatorname{Mod}^{\mathcal{I}},\left(\mid=\frac{\mathcal{I}}{\Sigma}\right)_{\Sigma \in\left|\operatorname{Sign}^{\mathcal{I}}\right|}\right)$ consists of

- a category $\operatorname{Sign}^{\mathcal{I}}$ whose objects are called signatures,

- a functor $\operatorname{Sen}^{\mathcal{I}}: \operatorname{Sign}^{\mathcal{I}} \rightarrow$ Set giving for each signature a set whose elements are called sentences over that signature,

- a functor $\operatorname{Mod}^{\mathcal{I}}:\left(\operatorname{Sign}^{\mathcal{I}}\right)^{\mathrm{op}} \rightarrow$ CAT, giving for each signature $\Sigma$ a category whose objects are called $\Sigma$-models, and whose arrows are called $\Sigma$-(model) homomorphisms, and

- a relation $\models=\frac{\mathcal{I}}{\Sigma} \subseteq\left|\operatorname{Mod}^{\mathcal{I}}(\Sigma)\right| \times \operatorname{Sen}^{\mathcal{I}}(\Sigma)$ for each $\Sigma \in\left|\operatorname{Sign}^{\mathcal{I}}\right|$, called the satisfaction relation,

such that for each morphism $\varphi: \Sigma \rightarrow \Sigma^{\prime} \in \operatorname{Sign}^{\mathcal{I}}$, the satisfaction condition

$$
M^{\prime} \models \sum_{\Sigma^{\prime}}^{\mathcal{I}} \operatorname{Sen}^{\mathcal{I}}(\varphi)(\rho) \text { if and only if } \operatorname{Mod}^{\mathcal{I}}(\varphi)\left(M^{\prime}\right) \models \stackrel{\mathcal{I}}{\Sigma} \rho
$$

holds for each $M^{\prime} \in\left|\operatorname{Mod}^{\mathcal{I}}\left(\Sigma^{\prime}\right)\right|$ and $\rho \in \operatorname{Sen}^{\mathcal{I}}(\Sigma)$.

Notation 2.1. It should be noted that the superscripts in $\operatorname{Sign}^{\mathcal{I}}, \operatorname{Sen}^{\mathcal{I}}, \operatorname{Mod}^{\mathcal{I}}, \models{ }^{\mathcal{I}}$ may be often omitted, and that given a signature morphism $\varphi, \operatorname{Sen}(\varphi)$ and $\operatorname{Mod}(\varphi)$ may usually be denoted by $\varphi$ and $\uparrow_{-} \uparrow_{\varphi}$, respectively.

In any institution as above we also use the following notations:

- for any $\mathcal{M} \subseteq|\operatorname{Mod}(\Sigma)|, \mathcal{M}^{*}$ denotes $\left\{\rho \in \operatorname{Sen}(\Sigma)|M|_{\Sigma} \rho\right.$ for each $\left.M \in \mathcal{M}\right\}$.

- for any $E \subseteq \operatorname{Sen}(\Sigma), E^{*}$ denotes $\left\{M \in|\operatorname{Mod}(\Sigma)| \mid M \models_{\Sigma} \rho\right.$ for each $\left.\rho \in E\right\}$.

- for any $E, E^{\prime} \subseteq \operatorname{Sen}(\Sigma), E \models E^{\prime}$ denotes $E^{*} \subseteq E^{\prime *}$.

- for any $E \subseteq \operatorname{Sen}(\Sigma), \operatorname{Mod}(\Sigma, E)$ is the full subcategory of $\operatorname{Mod}(\Sigma)$ whose objects are in $E^{*}$.

Myriads of logical systems from computing or from mathematical logic have been captured as institutions; in fact, the thesis underlying institution theory that anything that deserves the name 'logic' should be captured by Dfn. 2.1. Below we recall a few of them that will also be used in examples in our paper.

${ }^{1}$ Strictly speaking, this is only a 'quasi-category' living in a higher set-theoretic universe. 
Example 2.1 ( $F O L, A L G, E Q, R E L$ and $P L$ ). Let $F O L$ be the institution of first order logic with equality in its many sorted form.

Its signatures are triples $(S, F, P)$ consisting of

- a set of sort symbols $S$,

- a family $F=\left\{F_{w \rightarrow s} \mid w \in S^{*}, s \in S\right\}$ of sets of function symbols indexed by arities (for the arguments) and sorts (for the results), and

- a family $P=\left\{P_{w} \mid w \in S^{*}\right\}$ of sets of relation (predicate) symbols indexed by arities.

Signature morphisms map the three components in a compatible way. This means that a signature morphism $\varphi:(S, F, P) \rightarrow\left(S^{\prime}, F^{\prime}, P^{\prime}\right)$ consists of

- a function $\varphi^{\text {st }}: S \rightarrow S^{\prime}$,

- a family of functions $\varphi^{\mathrm{op}}=\left\{\varphi_{w \rightarrow s}^{\mathrm{op}}: F_{w \rightarrow s} \rightarrow F_{\varphi^{\mathrm{st}}(w) \rightarrow \varphi^{\mathrm{st}}(s)}^{\prime} \mid w \in S^{*}, s \in S\right\}$, and

- a family of functions $\varphi^{\mathrm{rl}}=\left\{\varphi_{w \rightarrow s}^{\mathrm{rl}}: P_{w} \rightarrow P_{\varphi^{\mathrm{st}}(w)}^{\prime} \mid w \in S^{*}, s \in S\right\}$.

Models $M$ for a signature $(S, F, P)$ are first order structures interpreting each sort symbol $s$ as a set $M_{s}$, each function symbol $\sigma$ as a function $M_{\sigma}$ from the product of the interpretations of the argument sorts to the interpretation of the result sort, and each relation symbol $\pi$ as a subset $M_{\pi}$ of the product of the interpretations of the argument sorts. By $|M|$ we denote $\left\{M_{s} \mid s \in S\right\}$ and we call it the universe of $M$ or the carrier set( $s$ ) of $M$. In order to avoid the existence of empty interpretations of the sorts, which may complicate unnecessarily our presentation, we assume that each signature has at least one constant (i.e. function symbol with empty arity) for each sort. A model homomorphism $h: M \rightarrow M^{\prime}$ is an indexed family of functions $\left\{h_{s}: M_{s} \rightarrow M_{s}^{\prime}\right\}_{s \in S}$ such that

- $h$ is an $(S, F)$-algebra homomorphism $M \rightarrow M^{\prime}$, i.e., $h_{s}\left(M_{\sigma}(m)\right)=M_{\sigma}^{\prime}\left(h_{w}(m)\right)$ for each $\sigma \in$ $F_{w \rightarrow s}$ and each $m \in M_{w}$, and

- $h_{w}(m) \in M_{\pi}^{\prime}$ if $m \in M_{\pi}$ (i.e. $h_{w}\left(M_{\pi}\right) \subseteq M_{\pi}^{\prime}$ ) for each relation $\pi \in P_{w}$ and each $m \in M_{w}$.

where $h_{w}: M_{w} \rightarrow M_{w}^{\prime}$ is the canonical component-wise extension of $h$, i.e. $h_{w}\left(m_{1}, \ldots, m_{n}\right)=\left(h_{s_{1}}\left(m_{1}\right), \ldots, h_{s_{n}}\left(m_{n}\right)\right)$ for $w=s_{1} \ldots s_{n}$ and $m_{i} \in M_{s_{i}}$ for $1 \leq i \leq n$.

For each signature morphism $\varphi: \Sigma \rightarrow \Sigma^{\prime}$, the reduct $M^{\prime} \uparrow_{\varphi}$ of a $\Sigma^{\prime}$-model $M^{\prime}$ is defined by $\left(M^{\prime} \uparrow_{\varphi}\right)_{x}=$ $M_{\varphi(x)}^{\prime}$ for each sort, function, or relation symbol $x$ from the domain signature of $\varphi$.

Sentences are the usual first order sentences built from equational and relational atoms by iterative application of Boolean connectives and first order quantifiers. Sentence translations along signature morphisms just rename the sorts, function, and relation symbols according to the respective signature morphisms. They can be formally defined by induction on the structure of the sentences. While the induction step is straightforward for the case of the Boolean connectives it needs a bit of attention for the case of the quantifiers. For any signature morphism $\varphi:(S, F, P) \rightarrow\left(S^{\prime}, F^{\prime}, P^{\prime}\right)$,

$$
\operatorname{Sen}^{F O L}(\varphi)((\forall X) \rho)=\left(\forall X^{\varphi}\right) \operatorname{Sen}^{F O L}\left(\varphi^{\prime}\right)(\rho)
$$

for each finite block $X$ of variables for $(S, F, P)$. The variables need to be disjoint from the constants of the signature, also we have to ensure that $\mathrm{Sen}^{F O L}$ thus defined is functorial indeed and that there is no overloading of variables (which in certain situations would cause a failure of the Satisfaction Condition). These may be formally achieved by considering that a variable for $(S, F, P)$ is a triple of the form $(x, s,(S, F, P))$ where $x$ is the name of the variable and $s \in S$ is the sort of the variable and that two 
different variables in $X$ have different names. We often abbreviate variables $(x, s,(S, F, P))$ by their name $x$. Then we let $(S, F+X, P)$ be the extension of $(S, F, P)$ such that $(F+X)_{w \rightarrow s}=F_{w \rightarrow s}$ when $w$ is non-empty and $(F+X)_{\rightarrow s}=F_{\rightarrow s} \cup\{(x, s,(S, F, P)) \mid(x, s,(S, F, P)) \in X\}$ and we let $\varphi^{\prime}:(S, F+X, P) \rightarrow\left(S^{\prime}, F^{\prime}+X^{\varphi}, P^{\prime}\right)$ be the canonical extension of $\varphi$ that maps each variable $(x, s,(S, F, P))$ to $\left(x, \varphi(s),\left(S^{\prime}, F^{\prime}, P^{\prime}\right)\right)$.

The satisfaction of sentences by models is the usual Tarskian satisfaction defined inductively on the structure of the sentences.

The institution $A L G$ is obtained from $F O L$ by discarding the relational symbols and their corresponding interpretations in models. The institution $E Q$ is defined as the sub-institution of $A L G$ where the sentences are just universally quantified equations $(\forall X) t=t^{\prime}$. The institution $R E L$ is the sub-institution of singlesorted first-order logic with signatures having only constants and relational symbols.

The institution $P L$ (of propositional logic) is the fragment of $F O L$ determined by signatures with empty sets of sort symbols.

Example 2.2 $(P A)$. Here we consider the institution $P A$ of partial algebra as employed by the specification language CASL [5].

A partial algebraic signature is a tuple $(S, T F, P F)$, where $T F$ is a family of sets of total function symbols and $P F$ is a family of sets of partial function symbols such that $T F_{w \rightarrow s} \cap P F_{w \rightarrow s}=\emptyset$ for each arity $w$ and each sort $s$. In order to avoid empty carriers, like in the case of $F O L$, we assume there exists at least one total constant for each sort. Signature morphisms map the three components in a compatible way.

A partial algebra is just like an ordinary algebra (i.e. a $F O L$ model without relations) but interpreting the function symbols of $P F$ as partial rather than total functions. A partial algebra homomorphism $h: A \rightarrow B$ is a family of (total) functions $\left\{h_{s}: A_{s} \rightarrow B_{s} \mid s \in S\right\}$ indexed by the set of sorts $S$ of the signature such that $h_{s}\left(A_{\sigma}(a)\right)=B_{\sigma}\left(h_{w}(a)\right)$ for each function symbol $\sigma \in T F_{w \rightarrow s} \cup P F_{w \rightarrow s}$ and each string of arguments $a \in A_{w}$ for which $A_{\sigma}(a)$ is defined.

The sentences have three kinds of atoms: definedness $\operatorname{df}(t)$, strong equality $t \stackrel{s}{=} t^{\prime}$, and existence equality $t \stackrel{e}{=} t^{\prime}$. The definedness $\operatorname{df}(t)$ of a term $t$ holds in a partial algebra $A$ when the interpretation $A_{t}$ of $t$ is defined. The strong equality $t \stackrel{s}{=} t^{\prime}$ holds when both terms are undefined or both of them are defined and are equal. The existence equality $t \stackrel{e}{=} t^{\prime}$ holds when both terms are defined and are equal. ${ }^{2}$ The sentences are formed from these atoms by Boolean connectives and quantifications over total variables (i.e variables that are always defined).

Recall from $[14,34]$ :

Definition 2.2 (Internal logic). An institution $\mathcal{I}$ has (semantic) conjunctions when for each signature $\Sigma$ and any $\Sigma$-sentences $e_{1}$ and $e_{2}$ there exists a $\Sigma$-sentence e such that $e^{*}=e_{1}^{*} \cap e_{2}^{*}$. Usually e is denoted by $e_{1} \wedge e_{2}$.

I has (semantic) implications when for each $e_{1}$ and $e_{2}$ as above there exists e such that $e^{*}=(\operatorname{Mod}(\Sigma)-$ $\left.e_{1}^{*}\right) \cup e_{2}^{*}$. Usually e is denoted $e_{1} \Rightarrow e_{2}$.

$\mathcal{I}$ has (semantic) existential $\mathcal{D}$-quantifications for a class $\mathcal{D}$ of signature morphisms when for each $\left(\chi: \Sigma \rightarrow \Sigma^{\prime}\right) \in \mathcal{D}$ when for each $\Sigma^{\prime}$-sentence $e^{\prime}$ there exists a $\Sigma$-sentence e such that $e^{*}=\operatorname{Mod}(\chi)\left(e^{*}\right)$. Usually e is denoted $(\exists \chi) e^{\prime}$.

In the same style we may extend this list also to other semantic Boolean connectives such as disjunction $(\vee)$, negation $(\neg)$, equivalence $(\Leftrightarrow)$ and to semantic universal quantifications $\left((\forall \chi) e^{\prime}\right)$.

\footnotetext{
${ }^{2}$ Notice that $\mathrm{df}(t)$ is equivalent to $t \stackrel{e}{=} t$ and that $t \stackrel{s}{=} t^{\prime}$ is equivalent to $\left(t \stackrel{e}{=} t^{\prime}\right) \vee\left(\neg \mathrm{df}(t) \wedge \neg \mathrm{df}\left(t^{\prime}\right)\right)$.
} 
We recall the notions of amalgamation and quantification space that are crucial for what follows. The former is intensely used in institution theory, whereas the latter concept was first introduced in [15] (but without a name). The respective definitions below represent a slight adaptation of the definitions from the literature to the needs of this paper; in this form Dfn. 2.3 and 2.5 have appeared in [30].

Definition 2.3 (Amalgamation property). Given any functor $\mathrm{Mod}: \mathrm{Sign}^{\mathrm{op}} \rightarrow$ CAT a commuting square of signature morphisms

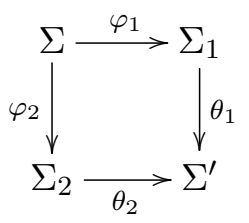

is a weak amalgamation square for Mod if and only if, for each $\Sigma_{1}$-model $M_{1}$ and $\Sigma_{2}$-model $M_{2}$ such that $\operatorname{Mod}\left(\varphi_{1}\right)\left(M_{1}\right)=\operatorname{Mod}\left(\varphi_{2}\right)\left(M_{2}\right)$, there exists a $\Sigma^{\prime}$-model $M^{\prime}$ such that $\operatorname{Mod}\left(\theta_{1}\right)\left(M^{\prime}\right)=M_{1}$ and $\operatorname{Mod}\left(\theta_{2}\right)\left(M^{\prime}\right)=M_{2}$. When $M^{\prime}$ is required to be unique, the square is called amalgamation square. The model $M^{\prime}$ is called an amalgamation of $M_{1}$ and $M_{2}$ and when it is unique it is denoted by $M_{1} \otimes_{\varphi_{1}, \varphi_{2}} M_{2}$.

We say that an institution $\mathcal{I}$ has the (weak) amalgamation property when each pushout square of signature morphisms is a (weak) amalgamation square for the model functor $\operatorname{Mod}^{\mathcal{I}}$.

Most of the institutions formalizing conventional or non-conventional logics have the amalgamation property $[14,18]$. These include our examples $F O L, A L G, P L, R E L, P A$.

Definition 2.4. A sub-functor $\mathrm{Mod}^{\prime} \subseteq \mathrm{Mod}: \operatorname{Sign}^{\mathrm{op}} \rightarrow$ CAT reflects (weak) amalgamation when each pushout square in Sign that is a (weak) amalgamation square for Mod is a (weak) amalgamation square for Mod' $^{\prime}$ too.

Definition 2.5 (Quantification space). For any category Sign a subclass of arrows $\mathcal{D} \subseteq$ Sign is called a quantification space if, for any $\left(\chi: \Sigma \rightarrow \Sigma^{\prime}\right) \in \mathcal{D}$ and $\varphi: \Sigma \rightarrow \Sigma_{1}$, there is a designated pushout

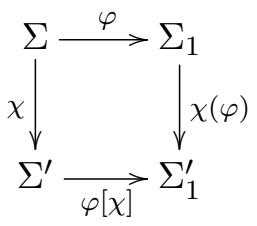

with $\chi(\varphi) \in \mathcal{D}$ and such that the 'horizontal' composition of such designated pushouts is again a designated pushout, i.e. for the pushouts in the following diagram

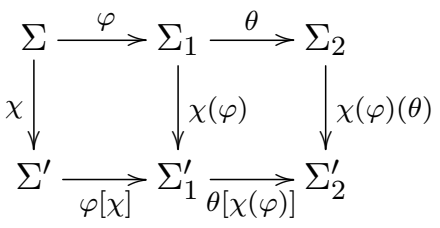

$\varphi[\chi] ; \theta[\chi(\varphi)]=(\varphi ; \theta)[\chi]$ and $\chi(\varphi)(\theta)=\chi(\varphi ; \theta)$, and such that $\chi\left(1_{\Sigma}\right)=\chi$ and $1_{\Sigma}[\chi]=1_{\Sigma^{\prime}}$.

We say that a quantification space $\mathcal{D}$ for $\operatorname{Sign}$ is adequate for a functor $\operatorname{Mod}: \operatorname{Sign}^{\text {op }} \rightarrow$ CAT when the designated pushouts mentioned above are weak amalgamation squares for Mod. 
Example 2.3. Within the context of Ex. 2.1 above, the signature extensions $\chi:(S, F, P) \hookrightarrow(S, F+X, P)$, where $X$ is a finite block of variables for $(S, F, P)$ constitute a quantification space for $\operatorname{Sign}^{F O L}$ that is also adequate for $\operatorname{Mod}^{F O L}$. Given signature morphism $\varphi:(S, F, P) \rightarrow\left(S_{1}, F_{1}, P_{1}\right)$, then

- $\chi(\varphi):\left(S_{1}, F_{1}, P_{1}\right) \hookrightarrow\left(S_{1}, F_{1}+X^{\varphi}, P_{1}\right)$ where $X^{\varphi}$ as defined in Ex. 2.1, and

$-\varphi[\chi]$ is the canonical extension of $\varphi$ that maps each $(x, s,(S, F, P))$ to $\left(x, \varphi^{\text {st }}(s),\left(S_{1}, F_{1}, P_{1}\right)\right)$ (it corresponds to $\varphi^{\prime}$ of Ex. 2.1).

It is easy to note that these define pushout squares fulfilling the properties of Dfn. 2.5. The adequacy for $\mathrm{Mod}^{F O L}$ follows from the fact that $\operatorname{Mod}^{F O L}$ preserves all finite limits (see [14]).

Other quantification spaces for $\mathrm{Sign}^{F O L}$ that are also adequate for $\mathrm{Mod}^{F O L}$ may be obtained as follows:

1. In the example above we consider infinite blocks of variables instead of finite ones.

2. We consider blocks of second order variables of the form $(x,(w, s),(S, F, P))$ (function variables) or of the form $(x, w,(S, F, P))$ (relation variables) where $w \in S^{*}$ and $s \in S$. Then to any block $X$ of second order variables it corresponds a signature extension $\chi:(S, F, P) \rightarrow\left(S, F+X^{\mathrm{op}}, P+X^{\mathrm{rl}}\right)$ where $X$ is split as $X^{\mathrm{op}} \cup X^{\mathrm{rl}}$ with $X^{\mathrm{op}}$ being the function variables and $X^{\mathrm{rl}}$ the relation variables, and where $F+X^{\mathrm{op}}$ and $P+X^{\mathrm{rl}}$ extend in the obvious way the definition of $F+X$ from Ex. 2.1.

Note that these definitions may also apply to $R E L$ and $A L G$. Similar definitions may also be developed in $P A$.

\section{Hybridized Institutions}

In this section we present the institution-independent construction of hybrid logics that has already been introduced in [30] as an extension of the previous work [19]. Here we include proofs that have been omitted in [30] and we refine the semantics of hybrid logics with the concept of constrained models that is treated fully abstractly as a sub-functor of the non-constrained model functor.

Let us consider an institution $\mathcal{I}=\left(\operatorname{Sign}^{\mathcal{I}}, \operatorname{Sen}^{\mathcal{I}}, \operatorname{Mod}^{\mathcal{I}},\left(\mid=\frac{\mathcal{I}}{\Sigma}\right)_{\Sigma \in\left|\operatorname{Sign}^{\mathcal{I}}\right|}\right)$ with a designated quantification space $\mathcal{D}^{\mathcal{I}} \subseteq \operatorname{Sign}^{\mathcal{I}}$. This will be referred to as the base institution. Below we introduce a method to enrich $\mathcal{I}$ with modalities and nominals, defining a suitable semantics for the enrichment. Moreover, it is shown that the outcome still defines a class of institutions, the so-called hybridizations of $\mathcal{I}$.

The category of $\mathcal{H} \mathcal{I}$-signatures:

The category of $\mathcal{I}$-hybrid signatures, denoted by $\operatorname{Sign}^{\mathcal{H I}}$, is defined as the following direct (cartezian) product of categories:

$$
\operatorname{Sign}^{\mathcal{H I}}=\operatorname{Sign}^{\mathcal{I}} \times \operatorname{Sign}^{R E L} .
$$

The $R E L$-signatures are denoted by (Nom, $\Lambda$ ), where Nom is a set of constants called nominals and $\Lambda$ is a set of relational symbols called modalities; $\Lambda_{n}$ stands for the set of modalities of arity $n$. For any

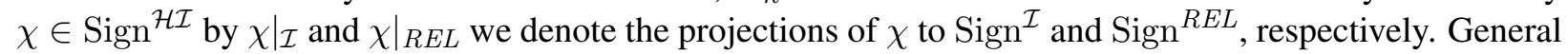
category theory entails:

Proposition 3.1. The projection $\operatorname{Sign}^{\mathcal{H} \mathcal{I}} \rightarrow \operatorname{Sign}^{\mathcal{I}}$ lifts small co-limits.

The existence of co-limits of signatures is one of the properties of institutions of key practical relevance for specification in-the-large (see [20]).

Corollary 3.1. $\operatorname{Sign}^{\mathcal{H I}}$ has all small co-limits that $\operatorname{Sign}^{\mathcal{I}}$ has. 


\section{$\mathcal{H} \mathcal{I}$-sentences:}

Let us fix a quantification space $\mathcal{D}^{\mathcal{H I}}$ for $\operatorname{Sign}{ }^{\mathcal{H I}}$ such that for each $\chi \in \mathcal{D}^{\mathcal{H I}}$ its projection $\left.\chi\right|_{\mathcal{I}}$ to $\operatorname{Sign}^{\mathcal{I}}$ belongs to $\mathcal{D}^{\mathcal{I}}$. The quantification space $\mathcal{D}^{\mathcal{H I}}$ is a parameter of the hybridization process. Whenever $\mathcal{D}^{\mathcal{H I}}$ consists of identities only we say the hybridization is quantifier-free. Note that a quantifier-free hybridization does not necessarily mean the absence of 'local' quantification, i.e. placed at the level of the base institution $\mathcal{I}$.

Let $\Delta=(\Sigma$, Nom, $\Lambda)$. The set of sentences $\operatorname{Sen}^{\mathcal{H I}}(\Delta)$ is the least set such that

- $\operatorname{Nom} \subseteq \operatorname{Sen}^{\mathcal{H} \mathcal{I}}(\Delta)$;

- $\operatorname{Sen}^{\mathcal{I}}(\Sigma) \subseteq \operatorname{Sen}^{\mathcal{H}}(\Delta)$;

- $\rho \star \rho^{\prime} \in \operatorname{Sen}^{\mathcal{H I}}(\Delta)$ for any $\rho, \rho^{\prime} \in \operatorname{Sen}^{\mathcal{H I}}(\Delta)$ and any $\star \in\{\vee, \wedge, \Rightarrow\}$,

- $\neg \rho \in \operatorname{Sen}^{\mathcal{H I}}(\Delta)$, for any $\rho \in \operatorname{Sen}^{\mathcal{H I}}(\Delta)$,

- $@_{i} \rho \in \operatorname{Sen}^{\mathcal{H I}}(\Delta)$ for any $\rho \in \operatorname{Sen}^{\mathcal{H I}}(\Delta)$ and $i \in \operatorname{Nom}$;

- $[\lambda]\left(\rho_{1}, \ldots, \rho_{n}\right),\langle\lambda\rangle\left(\rho_{1}, \ldots, \rho_{n}\right) \in \operatorname{Sen}^{\mathcal{H I}}(\Delta)$, for any $\lambda \in \Lambda_{n+1}, \rho_{i} \in \operatorname{Sen}^{\mathcal{H I}}(\Delta), i \in\{1, \ldots, n\}$;

- $(\forall \chi) \rho,(\exists \chi) \rho \in \operatorname{Sen}^{\mathcal{H I}}(\Delta)$, for any $\rho \in \operatorname{Sen}^{\mathcal{H I}}\left(\Delta^{\prime}\right)$ and $\chi: \Delta \rightarrow \Delta^{\prime} \in \mathcal{D}^{\mathcal{H} \mathcal{I}}$;

Translations of $\mathcal{H} \mathcal{I}$-sentences:

Let $\varphi=\left(\varphi_{\mathrm{Sig}}, \varphi_{\mathrm{Nom}}, \varphi_{\mathrm{MS}}\right):(\Sigma, \operatorname{Nom}, \Lambda) \rightarrow\left(\Sigma^{\prime}, \mathrm{Nom}^{\prime}, \Lambda^{\prime}\right)$ be a morphism of $\mathcal{H} \mathcal{I}$-signatures.

The translation $\operatorname{Sen}^{\mathcal{H I}}(\varphi)$ is defined as follows:

- $\operatorname{Sen}^{\mathcal{H I}}(\varphi)(i)=\varphi_{\mathrm{Nom}}(i)$;

- $\operatorname{Sen}^{\mathcal{H} \mathcal{I}}(\varphi)(\rho)=\operatorname{Sen}^{\mathcal{I}}\left(\varphi_{\mathrm{Sig}}\right)(\rho)$ for any $\rho \in \operatorname{Sen}^{\mathcal{I}}(\Sigma)$;

- $\operatorname{Sen}^{\mathcal{H I}}(\varphi)\left(\rho \star \rho^{\prime}\right)=\operatorname{Sen}^{\mathcal{H I}}(\varphi)(\rho) \star \operatorname{Sen}^{\mathcal{H I}}(\varphi)\left(\rho^{\prime}\right), \star \in\{\vee, \wedge, \Rightarrow\}$;

- $\operatorname{Sen}^{\mathcal{H I}}(\varphi)(\neg \rho)=\neg \operatorname{Sen}^{\mathcal{H I}}(\varphi)(\rho)$;

- $\operatorname{Sen}^{\mathcal{H I}}(\varphi)\left(@_{i} \rho\right)=@_{\varphi_{\mathrm{Nom}}(i)} \operatorname{Sen}^{\mathcal{H I}}(\rho)$;

- $\operatorname{Sen}^{\mathcal{H I}}(\varphi)\left([\lambda]\left(\rho_{1}, \ldots, \rho_{n}\right)\right)=\left[\varphi_{\mathrm{MS}}(\lambda)\right]\left(\operatorname{Sen}^{\mathcal{H I}}\left(\rho_{1}\right), \ldots, \operatorname{Sen}^{\mathcal{H I}}\left(\rho_{n}\right)\right)$;

- $\operatorname{Sen}^{\mathcal{H I}}(\varphi)\left(\langle\lambda\rangle\left(\rho_{1}, \ldots, \rho_{n}\right)\right)=\left\langle\varphi_{\mathrm{MS}}(\lambda)\right\rangle\left(\operatorname{Sen}^{\mathcal{H I}}\left(\rho_{1}\right), \ldots, \operatorname{Sen}^{\mathcal{H I}}\left(\rho_{n}\right)\right)$;

- $\operatorname{Sen}^{\mathcal{H I}}(\varphi)((\forall \chi) \rho)=(\forall \chi(\varphi)) \operatorname{Sen}^{\mathcal{H I}}(\varphi[\chi])(\rho)$.

- $\operatorname{Sen}^{\mathcal{H I}}(\varphi)((\exists \chi) \rho)=(\exists \chi(\varphi)) \operatorname{Sen}^{\mathcal{H I}}(\varphi[\chi])(\rho)$.

The following result may be obtained by recursion on the structure of the sentences by straightforward calculations (omitted here), the part corresponding to the quantifiers relying crucially upon the properties expressed in Dfn. 2.5.

Proposition 3.2. $\operatorname{Sen}^{\mathcal{H I}}$ is a functor $\operatorname{Sign}^{\mathcal{H I}} \rightarrow$ Set.

$\mathcal{H} \mathcal{I}$-models:

The $(\Sigma$, Nom, $\Lambda)$-models are pairs $(M, R)$ where

- $R$ is a (Nom, $\Lambda$ )-model in $R E L$;

- $M$ is a function $|R| \rightarrow\left|\operatorname{Mod}^{\mathcal{I}}(\Sigma)\right|$. 
The carrier set $|R|$ forms the set of the states of $(M, R) ;\left\{R_{n} \mid n \in\right.$ Nom $\}$ represents the interpretations of the nominals Nom, whereas relations $\left\{R_{\lambda} \mid \lambda \in \Lambda_{n}, n \in \omega\right\}$ represent the interpretation of the modalities $\Lambda$. We denote $M(s)$ simply by $M_{s}$.

A $(\Sigma$, Nom, $\Lambda)$-model homomorphism $h:(M, R) \rightarrow\left(M^{\prime}, R^{\prime}\right)$ consists of a pair aggregating

- a (Nom, $\Lambda$ )-model homomorphism in $R E L, h_{s t}: R \rightarrow R^{\prime}$; i.e., a function $h_{s t}:|R| \rightarrow\left|R^{\prime}\right|$ such that for $i \in$ Nom, $R_{i}^{\prime}=h_{s t}\left(R_{i}\right)$; and, for any $s_{1}, \ldots, s_{n} \in|R|, \lambda \in \Lambda_{n}$, and $\left(s_{1}, \ldots, s_{n}\right) \in R_{\lambda}$, $\left(h_{s t}\left(s_{1}\right), \ldots, h_{s t}\left(s_{n}\right)\right) \in R_{\lambda}^{\prime}$.

- a natural transformation $h_{m o d}: M \Rightarrow M^{\prime} \circ h_{s t},{ }^{3}$ i.e. $h_{m o d}$ is a $|R|$-indexed family of $\Sigma$-model homomorphisms $h_{\text {mod }}=\left\{\left(h_{\text {mod }}\right)_{s}: M_{s} \rightarrow M_{h_{s t}(s)}^{\prime}|s \in| R \mid\right\}$. In the text sometimes we may abbreviate $\left(h_{m o d}\right)_{s}$ by $h_{s}$.

The composition of $\mathcal{H} \mathcal{I}$-model homomorphisms is defined canonically as

$$
h ; h^{\prime}=\left(h_{s t} ; h_{s t}^{\prime}, h_{m o d} ;\left(h_{m o d}^{\prime} \circ h_{s t}\right)\right) .
$$

Fact 3.1. Let $\Delta$ be any $\mathcal{H} \mathcal{I}$-signature. Then $\Delta$-models together with their homomorphisms constitute a category, denoted $\operatorname{Mod}^{\mathcal{H I}}(\Delta)$.

\section{Reducts of $\mathcal{H I}$-models:}

Let $\Delta=(\Sigma$, Nom, $\Lambda)$ and $\Delta^{\prime}=\left(\Sigma^{\prime}, \mathrm{Nom}^{\prime}, \Lambda^{\prime}\right)$ be two $\mathcal{H} \mathcal{I}$-signatures, $\varphi=\left(\varphi_{\mathrm{Sig}}, \varphi_{\mathrm{Nom}}, \varphi_{\mathrm{MS}}\right)$ a morphism between $\Delta$ and $\Delta^{\prime}$ and $\left(M^{\prime}, R^{\prime}\right)$ a $\Delta^{\prime}$-model. The reduct of $\left(M^{\prime}, R^{\prime}\right)$ along $\varphi$, denoted by $\operatorname{Mod}^{\mathcal{H I}}(\varphi)\left(M^{\prime}, R^{\prime}\right)$, is the $\Delta$-model $(M, R)$ such that

- $R$ is the $\left(\varphi_{\mathrm{Nom}}, \varphi_{\mathrm{MS}}\right)$-reduct of $R^{\prime}$; i.e.

- $|R|=\left|R^{\prime}\right|$

- for any $n \in \operatorname{Nom}, R_{n}=R_{\varphi_{\text {Nom }}(n)}^{\prime}$;

- for any $\lambda \in \Lambda, R_{\lambda}=R_{\varphi_{\mathrm{MS}}(\lambda)}^{\prime}$;

and

- for any $s \in|R|, M_{s}=\operatorname{Mod}^{\mathcal{I}}\left(\varphi_{\mathrm{Sig}}\right)\left(M_{s}^{\prime}\right)$.

For any $h^{\prime}:\left(M_{1}^{\prime}, R_{1}^{\prime}\right) \rightarrow\left(M_{2}^{\prime}, R_{2}^{\prime}\right)$ its $\varphi$-reduct is $\left(h_{s t}^{\prime}, h_{m o d}^{\prime} \operatorname{Mod}^{\mathcal{I}}(\varphi)\right)$.

Fact 3.2. $\operatorname{Mod}^{\mathcal{H I}}$ is a functor $\left(\operatorname{Sign}^{\mathcal{H I}}\right)^{\mathrm{op}} \rightarrow \mathbf{C A T}$.

Theorem 3.1. A pushout square of $\mathcal{H} \mathcal{I}$-signature morphisms is a (weak) amalgamation square (for $\mathrm{Mod}^{\mathcal{H} \mathcal{I}}$ ) if the underlying square of signature morphisms in $\mathcal{I}$ is a (weak) amalgamation square.

Proof. Given a pushout of $\mathcal{H} \mathcal{I}$-signature morphisms like in Dfn. 2.3, the amalgamation $\left(M^{\prime}, R^{\prime}\right)$ of $\left(M_{1}, R_{1}\right)$ and $\left(M_{2}, R_{2}\right)$ is defined as follows:

- $R^{\prime}$ is an amalgamation of $R_{1}$ and $R_{2}$ is $R E L$; this is because the projection functor $\operatorname{Sign}^{\mathcal{H I}}=\operatorname{Sign}^{\mathcal{I}} \times$ $\operatorname{Sign}^{R E L} \rightarrow \operatorname{Sign}^{R E L}$ preserves pushouts, hence the underlying square of signature morphisms in $R E L$ is pushout.

\footnotetext{
${ }^{3}$ Where $M^{\prime} \circ h_{s t}$ is an alternative notation for $h_{s t} ; M^{\prime}$, more convenient in this context.
} 
- Let $|R|$ be the carrier set of $R_{1}, R_{2}$ and $R$ (by definition of reduct note all of them share the same carrier set). Then $M^{\prime}:|R| \rightarrow\left|\operatorname{Mod}^{\mathcal{I}}\left(\Sigma^{\prime}\right)\right|$ is the function determined by the (weak) pullback property of the result of applying the functor $\left|\operatorname{Mod}^{\mathcal{I}}(-)\right|$ to the underlying square of signature morphisms in $\mathcal{I}$.

Corollary 3.2. If $\mathcal{D}^{\mathcal{I}}$ is adequate for $\operatorname{Mod}^{\mathcal{I}}$ then $\mathcal{D}^{\mathcal{H I}}$ is adequate for $\operatorname{Mod}^{\mathcal{H I}}$.

Proof. Any designated square $S q$ in the quantification space $\mathcal{D}^{\mathcal{H I}}$ is a pushout by definition and since the projection functor $\operatorname{Sign}^{\mathcal{H} \mathcal{I}}=\operatorname{Sign}^{\mathcal{I}} \times \operatorname{Sign}^{R E L} \rightarrow \operatorname{Sign}^{\mathcal{I}}$ preserves pushouts it follows that the underlying square $S q_{0}$ of $S q$ consisting of $\mathcal{I}$ signature morphisms is also a pushout. Because of this and also because $\left.\mathcal{D}^{\mathcal{H I}}\right|_{\mathcal{I}} \subseteq \mathcal{D}^{\mathcal{I}}$ it follows that $S q_{0}$ is isomorphic (as pushout co-cone) to a designated square in the quantification space $\mathcal{D}^{\mathcal{I}}$, which by the adequacy property of $\mathcal{D}^{\mathcal{I}}$ with respect to $\operatorname{Mod}^{\mathcal{I}}$ is a weak amalgamation square. Hence $S q_{0}$ is a weak amalgamation square, which by Thm. 3.1 implies that $S q$ is adequate for $\operatorname{Mod}^{\mathcal{H I}}$.

\section{Constrained models:}

Often the semantics of modal and hybrid logics may include various additional constraints on the models. The following definition captures abstractly this situation.

Definition 3.1. $A$ constrained $\mathcal{H} \mathcal{I}$-model functor is a sub-functor $\operatorname{Mod}^{\prime} \subseteq \operatorname{Mod}^{\mathcal{H I}}$ such that it reflects weak amalgamation. The models in $\mathrm{Mod}^{\prime}$ are called constrained $\mathcal{H} \mathcal{I}$-models.

Informally, the meaning of Dfn. 3.1 is that in the case of pushout squares of signature morphisms any two constrained models that can be amalgamated admit a constrained amalgamation.

The following result, which is an immediate consequence of Cor. 3.2, Dfn. 3.1 and Dfn. 2.4, applies often in concrete situations, including all the examples in our paper.

Corollary 3.3. If $\mathcal{D}^{\mathcal{I}}$ is adequate for $\operatorname{Mod}^{\mathcal{I}}$ then $\mathcal{D}^{\mathcal{H I}}$ is adequate for any constrained $\mathcal{H I}$-model functor Mod'.

The Satisfaction Relation:

Given a constrained model functor $\operatorname{Mod}^{\prime} \subseteq \operatorname{Mod}^{\mathcal{H I}}$, for any $(M, R) \in\left|\operatorname{Mod}^{\prime}(\Sigma, \operatorname{Nom}, \Lambda)\right|$ and for any $s \in|R|$ we define:

- $(M, R) \models^{s} i$ iff $R_{i}=s$; when $i \in$ Nom,

- $(M, R) \models^{s} \rho$ iff $M_{s} \models^{\mathcal{I}} \rho$; when $\rho \in \operatorname{Sen}^{\mathcal{I}}(\Sigma)$,

- $(M, R) \models^{s} \rho \vee \rho^{\prime}$ iff $(M, R) \models^{s} \rho$ or $(M, R) \models^{s} \rho^{\prime}$,

- $(M, R) \models^{s} \rho \wedge \rho^{\prime}$ iff $(M, R) \models^{s} \rho$ and $(M, R) \models^{s} \rho^{\prime}$,

- $(M, R) \models^{s} \rho \Rightarrow \rho^{\prime}$ iff $(M, R) \models^{s} \rho$ implies that $(M, R) \models^{s} \rho^{\prime}$,

- $(M, R) \models^{s} \neg \rho$ iff $(M, R) \quad \chi^{s} \rho$,

- $(M, R) \models^{s} @_{j} \rho$ iff $(M, R) \models^{R_{j}} \rho$,

- $(M, R) \models^{s}[\lambda]\left(\rho_{1}, \ldots \rho_{n}\right)$ iff $(M, R) \models^{s_{i}} \rho_{i}$ for some $1 \leq i \leq n$ for any $\left(s, s_{1}, \ldots, s_{n}\right) \in R_{\lambda}$, $\lambda \in \Lambda_{n+1}$, 
- $(M, R) \models=^{s}\langle\lambda\rangle\left(\rho_{1}, \ldots \rho_{n}\right)$ iff $(M, R) \models^{s_{i}} \rho_{i}$ for any $1 \leq i \leq n$ for some $\left(s, s_{1}, \ldots, s_{n}\right) \in R_{\lambda}$, $\lambda \in \Lambda_{n+1}$,

- $(M, R) \models^{s}(\forall \chi) \rho$ iff $\left(M^{\prime}, R^{\prime}\right) \models^{s} \rho$ for any $\left(M^{\prime}, R^{\prime}\right)$ such that $\operatorname{Mod}^{\prime}(\chi)\left(M^{\prime}, R^{\prime}\right)=(M, R)$, and

- $(M, R) \models^{s}(\exists \chi) \rho$ iff $\left(M^{\prime}, R^{\prime}\right) \models^{s} \rho$ for some $\left(M^{\prime}, R^{\prime}\right)$ such that $\operatorname{Mod}^{\prime}(\chi)\left(M^{\prime}, R^{\prime}\right)=(M, R)$.

We write $(M, R) \models \rho$ iff $(M, R) \models{ }^{s} \rho$ for any $s \in|R|$.

\section{The Satisfaction Condition:}

Theorem 3.2. Assume $\mathcal{D}^{\mathcal{I}}$ is adequate for $\mathrm{Mod}^{\mathcal{I}}$. Let $\Delta=(\Sigma$, Nom, $\Lambda)$ and $\Delta^{\prime}=\left(\Sigma^{\prime}, \mathrm{Nom}^{\prime}, \Lambda^{\prime}\right)$ be two $\mathcal{H} \mathcal{I}$-signatures and $\varphi: \Delta \rightarrow \Delta^{\prime}$ a morphism of signatures. Given a constrained model functor $\operatorname{Mod}^{\prime} \subseteq$ $\operatorname{Mod}^{\mathcal{H I}}$, for any $\rho \in \operatorname{Sen}^{\mathcal{H I}}(\Delta),\left(M^{\prime}, R^{\prime}\right) \in\left|\operatorname{Mod}^{\prime}\left(\Delta^{\prime}\right)\right|$, and $s \in|R|$

$$
\operatorname{Mod}^{\prime}(\varphi)\left(M^{\prime}, R^{\prime}\right) \models^{s} \rho \text { if and only if }\left(M^{\prime}, R^{\prime}\right) \models^{s} \operatorname{Sen}^{\mathcal{H I}}(\varphi)(\rho) \text {. }
$$

Proof. Let us denote $\operatorname{Mod}^{\prime}\left(M^{\prime}, R^{\prime}\right)=\operatorname{Mod}^{\mathcal{H I}}\left(M^{\prime}, R^{\prime}\right)$ by $(M, R)$. The proof is by recursion on the structure of the sentence $\rho$ :

1. $\rho=i$ for some $i \in$ Nom:

$$
\begin{array}{rll}
(M, R) \models^{s} i & \text { iff } R_{i}=s & \text { (by definition of } \left.\models^{s}\right) \\
& \text { iff }\left(M^{\prime}, R^{\prime}\right) \models^{s} \varphi_{\text {Nom }}(i) & \text { (by definition of the reduct, } \left.R_{\varphi_{\text {Nom }}(i)}^{\prime}=R_{i}\right) \\
& \text { iff }\left(M^{\prime}, R^{\prime}\right) \models^{s} \operatorname{Sen}^{\mathcal{H I}}(\varphi)(i) & \text { (by definition of } \left.\operatorname{Sen}^{\mathcal{H I}}(\varphi)\right) .
\end{array}
$$

2. $\rho \in \operatorname{Sen}^{\mathcal{I}}(\Sigma)$ :

$$
\begin{aligned}
& (M, R) \models^{s} \rho \quad \text { iff } \operatorname{Mod}^{\mathcal{I}}\left(\varphi_{\text {Sig }}\right)\left(M_{s}^{\prime}\right)=M_{s} \models^{\mathcal{I}} \rho \quad\left(\text { by definition of } \models^{s}\right) \\
& \text { iff } M_{s}^{\prime}=\operatorname{Sen}^{\mathcal{I}}\left(\varphi_{\operatorname{Sig}}\right)(\rho) \quad \text { (by the Satisfaction Condition in } \mathcal{I} \text { ) } \\
& \text { iff } \left.\left(M^{\prime}, R^{\prime}\right) \models^{s} \operatorname{Sen}^{\mathcal{I}}\left(\varphi_{\operatorname{Sig}}\right)(\rho) \quad \text { (by definition of } \models^{s}\right) \\
& \text { iff }\left(M^{\prime}, R^{\prime}\right) \models{ }^{s} \operatorname{Sen}^{\mathcal{H I}}(\varphi)(\rho) \quad \text { (by definition of } \operatorname{Sen}^{\mathcal{H I}}(\varphi) \text { ). }
\end{aligned}
$$

3. $\rho=\xi \vee \xi^{\prime}$ for some $\xi, \xi^{\prime} \in \operatorname{Sen}^{\mathcal{H I}}(\Delta)$ :

$$
\begin{aligned}
& (M, R) \models^{s} \xi \vee \xi^{\prime} \quad \text { iff }(M, R) \models^{s} \xi \text { or }(M, R) \models^{s} \xi^{\prime} \\
& \text { iff }\left(M^{\prime}, R^{\prime}\right) \models^{s} \operatorname{Sen}^{\mathcal{H I}}(\varphi)(\xi) \text { or }\left(M^{\prime}, R^{\prime}\right) \models^{s} \operatorname{Sen}^{\mathcal{H I}}(\varphi)\left(\xi^{\prime}\right) \quad \text { (by induction hypothesis) } \\
& \text { iff }\left(M^{\prime}, R^{\prime}\right) \models{ }^{s} \operatorname{Sen}^{\mathcal{H I}}(\varphi)\left(\xi \vee \xi^{\prime}\right) \\
& \text { (by definition of } \models^{s} \text { ). }
\end{aligned}
$$

The proofs for the cases when $\rho=\xi \wedge \xi^{\prime}, \rho=\xi \Rightarrow \xi^{\prime}, \rho=\neg \xi$, etc. are analogous.

4. $\rho=@_{i} \xi$ for some $\xi \in \operatorname{Sen}^{\mathcal{H I}}(\Delta), i \in$ Nom:

$$
\begin{array}{rll}
(M, R)=^{s} @_{i} \xi & \text { iff }(M, R) \models{ }^{R_{i}} \xi & \text { (by definition of } \models^{s} \text { ) } \\
& \text { iff }\left(M^{\prime}, R^{\prime}\right) \models{ }^{R_{i}} \operatorname{Sen}^{\mathcal{H I}}(\varphi)(\xi) & \text { (by induction hypothesis) } \\
& \text { iff }\left(M^{\prime}, R^{\prime}\right) \models{ }^{R^{\prime}}{ }_{\varphi_{\mathrm{Nom}}}(i) \operatorname{Sen}^{\mathcal{H I}}(\varphi)(\xi) & \text { (since by reduct definition, } \left.R_{i}=R_{\varphi_{\mathrm{Nom}}(i)}^{\prime}\right) \\
& \text { iff }\left(M^{\prime}, R^{\prime}\right) \models^{s} @_{\varphi_{\mathrm{Nom}}(i)} \operatorname{Sen}^{\mathcal{H I}}(\varphi)(\xi) & \text { (by definition of satisfaction for @) } \\
& \text { iff }\left(M^{\prime}, R^{\prime}\right) \models^{s} \operatorname{Sen}^{\mathcal{H I}}(\varphi)\left(@_{i} \xi\right) & \text { (by definition of } \left.\operatorname{Sen}^{\mathcal{H I}}(\varphi)\right) .
\end{array}
$$


5. $\rho=[\lambda]\left(\xi_{1}, \ldots, \xi_{n}\right)$, for some $\xi_{1}, \ldots, \xi_{n} \in \operatorname{Sen}^{\mathcal{H I}}(\Delta), \lambda \in \Lambda_{n+1}$ :

$$
\begin{aligned}
(M, R) \models^{s}[\lambda]\left(\xi_{1}, \ldots, \xi_{n}\right) \quad \text { iff } \quad & \text { for any }\left(s, s_{1}, \ldots, s_{n}\right) \in R_{\lambda},(M, R)=^{s_{i}} \xi_{i} \text { for some } 1 \leq i \leq n \\
& \text { (by definition of } \left.\models^{s}\right) \\
\text { iff } \quad & \left(M^{\prime}, R^{\prime}\right) \models^{s_{i}} \operatorname{Sen}^{\mathcal{H I}}(\varphi)\left(\xi_{i}\right) \text { for any }\left(s, s_{1}, \ldots, s_{n}\right) \in \varphi_{\mathrm{MS}}\left(R_{\lambda}^{\prime}\right) \text { and some } 1 \leq R_{\lambda} \\
& \text { (induction hypothesis and reduct definition entail } \left.R_{\lambda}=R_{\varphi_{\mathrm{MS}}(\lambda)}^{\prime}\right) \\
\text { iff } & \left(M^{\prime}, R^{\prime}\right) \models^{s}\left[\varphi_{\mathrm{MS}}(\lambda)\right] \operatorname{Sen}^{\mathcal{H I}}(\varphi)\left(\xi_{1}, \ldots, \xi_{n}\right) \\
& \left(\text { by definition of } \models^{s}\right) \\
\text { iff } & \left(M^{\prime}, R^{\prime}\right) \models^{s} \operatorname{Sen}^{\mathcal{H I}}(\varphi)\left([\lambda]\left(\xi_{1}, \ldots, \xi_{n}\right)\right) \\
& \left(\text { by definition of } \operatorname{Sen}^{\mathcal{H I}}(\varphi)\right) .
\end{aligned}
$$

The proof for the case when $\rho=\langle\lambda\rangle\left(\xi_{1}, \ldots, \xi_{n}\right)$, for some $\xi_{1}, \ldots, \xi_{n} \in \operatorname{Sen}^{\mathcal{H I}}(\Delta), \lambda \in \Lambda_{n+1}$ is analogous.

6. $\rho=\left(\forall \chi: \Delta \rightarrow \Delta_{1}\right) \xi$ :

$$
\begin{aligned}
& (M, R) \models^{s}(\forall \chi) \xi \quad \text { iff } \quad \text { for all }\left(M_{1}, R_{1}\right) \text { such that } \operatorname{Mod}^{\prime}(\chi)\left(M_{1}, R_{1}\right)=(M, R),\left(M_{1}, R_{1}\right) \models^{s} \xi \\
& \text { (by definition of satisfaction for }(\forall \chi) \xi) \\
& \text { iff for all }\left(M_{1}^{\prime}, R_{1}^{\prime}\right) \text { such that } \operatorname{Mod}^{\prime}(\chi(\varphi))\left(M_{1}^{\prime}, R_{1}^{\prime}\right)=\left(M^{\prime}, R^{\prime}\right) \text {, } \\
& \operatorname{Mod}^{\prime}(\varphi[\chi])\left(M_{1}^{\prime}, R_{1}^{\prime}\right) \models{ }^{s} \xi \text { (by Cor. 3.3, } \mathcal{D}^{\mathcal{H I}} \text { is adequate for } \operatorname{Mod}^{\prime} \text { ) } \\
& \text { iff for all }\left(M_{1}^{\prime}, R_{1}^{\prime}\right) \text { such that } \operatorname{Mod}^{\prime}(\chi(\varphi))\left(M_{1}^{\prime}, R_{1}^{\prime}\right)=\left(M^{\prime}, R^{\prime}\right) \text {, } \\
& \left(M_{1}^{\prime}, R_{1}^{\prime}\right) \models^{s} \operatorname{Sen}^{\mathcal{H I}}(\varphi[\chi])(\xi) \text { (by induction hypothesis) } \\
& \text { iff } \quad\left(M^{\prime}, R^{\prime}\right) \models^{s}(\forall \chi(\varphi)) \operatorname{Sen}^{\mathcal{H I}}(\varphi[\chi])(\xi) \\
& \text { (by definition of satisfaction for } \left.(\forall \chi(\varphi)) \operatorname{Sen}^{\mathcal{H I}}(\varphi[\chi])(\xi)\right) \\
& \text { iff } \quad\left(M^{\prime}, R^{\prime}\right) \models^{s} \operatorname{Sen}^{\mathcal{H I}}(\varphi)((\forall \chi) \xi) \\
& \text { (by definition of } \operatorname{Sen}^{\mathcal{H I}}(\varphi) \text { ). }
\end{aligned}
$$

The proof for the case when $\rho=\left(\exists \chi: \Delta \rightarrow \Delta_{1}\right) \xi$ is analogous.

Note that in the quantifier-free situation, i.e. when $\mathcal{D}^{\mathcal{H I}}$ is trivial, the adequacy assumption of Thm. 3.2 is not needed, and moreover the reflection condition for the constrained model functor is also not needed.

Corollary 3.4 (The Satisfaction Condition). $\left(\operatorname{Sign}^{\mathcal{H I}}, \operatorname{Sen}^{\mathcal{H I}}, \operatorname{Mod}^{\prime}, \models^{\prime}\right)$ is an institution.

Let us call the institution $\left(\operatorname{Sign}^{\mathcal{H I}}, \operatorname{Sen}^{\mathcal{H I}}, \operatorname{Mod}^{\prime}, \mid=\right)$ a hybridization of $\mathcal{I}$. The hybridization $\left(\operatorname{Sign}^{\mathcal{H I}}, \operatorname{Sen}^{\mathcal{H I}}, \operatorname{Mod}^{\mathcal{H I}}, \mid=\right.$ ), that does not constrain models, is denoted $\mathcal{H I}$ and is called the free hybridization of $\mathcal{I}$. Note that in general, because of the quantifiers, the satisfaction relation $\models^{\prime}$ of a hybridization with properly constrained models is not necessarily the restriction of $\models^{\mathcal{H}}$, the satisfaction relation of $\mathcal{H} \mathcal{I}$. Also hybridizations of institutions constitute an example of the general notion of stratified institution of [2].

\section{Base logic versus hybrid logic:}

In hybridized institutions, at the level of the sentences of the base institution we may have two sets of Boolean connectives, those of the hybridization and those of the base institution (when the base institution has them). The following simple result allows us to ignore the distinction between the Boolean connectives of a hybridization and those of the base institution. The result also states the general relationship between the quantification at the base and at the hybridized level. 
Fact 3.3. For any hybridization of $\mathcal{I}$, $\left(\operatorname{Sign}^{\mathcal{H I}}, \operatorname{Sen}^{\mathcal{H I}}, \operatorname{Mod}^{\prime} \subseteq \operatorname{Mod}^{\mathcal{H I}}, \mid=\right)$, let us denote the Boolean connectives and the quantifiers in the base institution $\mathcal{I}$ by $(\Theta, \ominus, \ominus, \ominus$, and $\oslash,(\ominus$, respectively. For any $(\Sigma, \operatorname{Nom}, \Lambda)$-model $(M, R)$, any $s \in|R|$, and any sentences $\rho, \rho^{\prime} \in \operatorname{Sen}^{\mathcal{I}}(\Sigma)$ of the base institution and for each $\chi \in \mathcal{D}$

$$
\begin{aligned}
& \text { - }(M, R) \models^{s} \rho \star \rho^{\prime} \text { iff }(M, R) \models^{s} \rho \circledast \rho^{\prime} \text { for } \star \in\{\wedge, \vee, \Rightarrow\} \text {, } \\
& -(M, R) \models{ }^{s} \neg \rho \text { iff }(M, R) \models^{s} \bigcirc \rho \text {, } \\
& \text { - }(M, R) \models^{s}(\forall \chi) \rho \text { implies }(M, R) \models^{s}\left(\forall\left(\chi, 1_{\text {Nom }}, 1_{\Lambda}\right)\right) \rho \text {, and } \\
& \text { - }(M, R) \models^{s}\left(\exists\left(\chi, 1_{\mathrm{Nom}}, 1_{\Lambda}\right)\right) \rho \text { implies }(M, R) \models^{s}(\Theta \chi) \rho \text {. }
\end{aligned}
$$

\section{Examples:}

A myriad of examples of hybridization may be generated from our definition above by considering various instances for the three parameters of our hybridization process: (1) the base institution $\mathcal{I}$, (2) the quantification space $\mathcal{D}^{\mathcal{H I}}$, and (3) the constrained models given by Mod'. We present only a few of them that will be used below in the paper as benchmark examples. Some of the examples below consider also sub-institutions determined by sub-categories of the category $\operatorname{Sign}^{\mathcal{H I}}$ of the $\mathcal{H} \mathcal{I}$-signatures; this does not imply any additional non-trivial technical considerations.

Example 3.1 $(\mathcal{H} P L)$. Applying the quantifier-free version of the hybridization method described above to $P L$ and fixing $\Lambda_{2}=\{\lambda\}$ and $\Lambda_{n}=\emptyset$ for each $n \neq 2$, we obtain the institution of the "standard" hybrid propositional logic (without state quantifiers): the category of signatures is $\operatorname{Sign}^{\mathcal{H} P L}=\operatorname{Set} \times$ Set with objects denoted by $(P$, Nom $)$ and morphisms by $\left(\varphi_{\mathrm{Sig}}, \varphi_{\mathrm{Nom}}\right)$; sentences are the usual hybrid propositional formulas, i.e., modal formulas closed by Boolean connectives, $[\lambda]$ denoted $\square,\langle\lambda\rangle$ denoted $\diamond$, and by the operator $@_{i}, i \in$ Nom; models consists of pairs $(M, R)$ where $R$ consists of a carrier set $|R|$, interpretations $R_{i} \in|R|$ for each $i \in$ Nom, and a binary relation $R_{\lambda} \subseteq|R| \times|R|$, and for each $s \in|R|, M_{s}$ is a propositional model, i.e., a function $M_{s}: P \rightarrow\{0,1\}$ which is equivalent to a subset $M_{s} \subseteq P$. Note that by virtue of Fact 3.3 we do not need to make a distinction between the Boolean connectives at the level of $P L$ and at the level of $\mathcal{H} P L$.

The $T, S 4$, and $S 5$ versions of hybrid propositional logic are obtained by constraining the models of $\mathcal{H} P L$ to those models $(M, R)$ for which $R_{\lambda}$ is reflexive, preorder, and equivalence, respectively.

When we relax to arbitrary sets of modalities $\Lambda$ rather than only $\lambda$, we obtain the "multi-modal hybrid propositional logic".

A challenging issue concerns finding suitable quantification spaces to capture versions of hybrid propositional logic. One choice is the quantifier-free version presented above in which $\mathcal{D}^{\mathcal{H} P L}$ consists only if identities. However, it would be interesting, along the hybridization process, to capture a quantifier such as $\mathbf{E}$, where $\mathbf{E} \rho$ means that " $\rho$ is true in some state of the model" [4]. Considering as a quantification space the extensions of signatures with nominal symbols, paves the way to express the following properties:

\begin{tabular}{|l|l|c|}
\hline$(M, R) \models^{s}((\forall i) i) \Leftrightarrow \rho$ & iff & $\rho$ is satisfied at $s$ iff $s$ is unique in $(M, R)$ \\
\hline$(M, R) \models^{s}(\exists i) @_{i} \rho$ & iff & $(M, R) \models \mathbf{E} \rho$ \\
\hline
\end{tabular}

A block of nominal variables $X$ for a $\mathcal{H} P L$ signature $(P, \mathrm{Nom})$ is a finite set of triples of the form $(x, P, \mathrm{Nom})$. Then $\mathcal{D}^{\mathcal{H} P L}$ may be defined as consisting of the signature extensions with blocks of nominal variables, i.e. $(P$, Nom $) \hookrightarrow(P, \operatorname{Nom} \cup X)$. For any signature morphism $\varphi:(P$, Nom $) \rightarrow\left(P^{\prime}, \mathrm{Nom}^{\prime}\right)$ and $X$ block of nominal variables for $(P$, Nom $)$ we define $X^{\varphi}=\left\{\left(x, P^{\prime}, \mathrm{Nom}^{\prime}\right) \mid(x, P\right.$, Nom $\left.) \in X\right\}$. Then $\chi(\varphi)$ is the extension $\left(P^{\prime}, \mathrm{Nom}^{\prime}\right) \hookrightarrow\left(P^{\prime}, \operatorname{Nom}^{\prime} \cup X^{\varphi}\right)$ and $\varphi[\chi]$ is the canonical extension of $\varphi$ that maps each $(x, P$, Nom $)$ to $\left(x, P^{\prime}\right.$, Nom $\left.^{\prime}\right)$. 
When we combine this quantification with the constraints T, S4, S5, etc., then we have to establish the adequacy condition for the constrained model sub-functor. However in this case this is almost trivial since we may consider $\mathcal{D}^{P L}$ (the quantification space at the level of the base institution) as being trivial and furthermore it is also immediate that the amalgamation of constrained models is still a constrained model.

Example 3.2 ( $\mathcal{H} F O L, \mathcal{H} E Q)$. Through the application of the hybridization method to $F O L$ by taking as a quantification space signature extensions both with $F O L$ variables and variables over nominals, one captures the state-variables quantification of first-order hybrid logic of [9]. Like in the case of $\mathcal{H} P L$, by virtue of the Fact 3.3 note that we do not need to make a distinction between the Boolean connectives at the level of FOL and those at the level of $\mathcal{H F O L}$. Moreover, because of the carriers of the FOL models are nonempty we may easily show that in this case the implications of Fact 3.3 about quantifiers may be turned into equivalences, hence it is also not necessary to distinguish between quantifiers at the base $F O L$ level and at the hybridized $\mathcal{H} F O L$ level.

Binding "state variables" to the point of evaluation highly increase the expressive power of an hybrid logic, which is enabled through the binder operator $\downarrow$ (e.g. [7, 9]). This may be achieved by taking $i$ extensions $\chi:(\Sigma$, Nom, $\Lambda) \hookrightarrow(\Sigma$, Nom $\uplus\{i\}, \Lambda)$ as a quantification space; in this case $(\downarrow i) \rho$ may be defined as $(\forall \chi)(i \Rightarrow \rho)$.

Let us also mention the quantifier-free hybridization of $E Q$. The resulting hybrid equational institution provides a suitable setting for specifying evolving systems in which each state is endowed with a specific algebra [29].

Example 3.3. Let $\mathcal{H} R E L^{\prime}$ be the hybridization of $R E L$ that constraints the models of $\mathcal{H} R E L$ to those models $(M, R)$ such that $\left\{M_{i}|i \in| R \mid\right\}$ share the same universe (underlying set) and the same interpretation of the constants. The sharing is also extended to model homomorphisms: for all $\mathcal{H} R E L^{\prime} \Delta$-models $(M, R)$ and $\left(M^{\prime}, R^{\prime}\right)$ a model homomorphism $h:(M, R) \rightarrow\left(M^{\prime}, R^{\prime}\right)$ in $\mathcal{H} R E L$ belongs to $\mathcal{H} R E L^{\prime}$ if and only if $h_{i}=h_{j}$ for all $i, j \in|R|$. It is rather easy to note that the amalgamation of models preserves the sharing, hence the reflection condition of Dfn. 3.1 is fulfilled.

$\mathcal{D}^{\mathcal{H} R E L^{\prime}}$ consists of the signature extensions with $F O L$ variables (for the states), with nominal variables (in the style of $\mathcal{D}^{\mathcal{H} P L}$ of Ex. 3.1) and with variables for modalities.

Note that like for $\mathcal{H} F O L$, in $\mathcal{H} R E L^{\prime}$ we also do not need to distinguish between the Boolean connectives and the quantifiers at the base and at the hybridized level.

Example 3.4. Let $\mathcal{H} P A^{\prime}$ be a hybridization of $P A$ that constraints the models of $\mathcal{H} P A$ to those models $(M, R)$ such that

- $\left\{M_{i}|i \in| R \mid\right\}$ share the same universes and the same interpretations of the total function symbols, i.e. for all $i, j \in|R|,\left(M_{i}\right)_{x}=\left(M_{j}\right)_{x}$ for any $x$ sort or total function symbol.

- for each $i, j \in|R|$, each partial function symbol $\sigma$ with arity $w$, and each $m \in\left(M_{i}\right)_{w}$, if both $\left(M_{i}\right)_{\sigma}(m)$ and $\left(M_{j}\right)_{\sigma}(m)$ are defined then $\left(M_{i}\right)_{\sigma}(m)=\left(M_{j}\right)_{\sigma}(m)$.

Like in Ex. 3.3 we also require that the model homomorphisms $h$ in $\mathcal{H} P A^{\prime}$ are such that $h_{i}=h_{j}$ for all $i, j \in|R|$. It is rather easy to note that the amalgamation of models preserves the sharing, hence the reflection condition of Dfn. 3.1 is fulfilled.

$\mathcal{D}^{\mathcal{H} P A^{\prime}}$ consists of the signature extensions with $P A$ variables (for the states), with nominal variables (in the style of $\mathcal{D}^{\mathcal{H} P L}$ of Ex. 3.1) and with variables for modalities.

Note that like for $\mathcal{H} F O L$ and $\mathcal{H} R E L^{\prime}$, in $\mathcal{H} P A^{\prime}$ we also do not need to distinguish between the Boolean connectives and the quantifiers at the base and at the hybridized level. 
Example 3.5. Let $\mathcal{H} A L G^{\prime}$ be a hybridization of $A L G$ that restricts the signatures to those in which $\Lambda$ has only two binary modality symbols $\leq$ and $\lambda$, and that constraints the models to those models $(M, R)$ for which $\left(|R|, R_{<}\right)$is a complete lattice and $M_{i}$ is a sub-algebra of $M_{j}$ whenever $(i, j) \in R_{<}$. Model homomorphisms $h:(M, R) \rightarrow\left(M^{\prime}, R^{\prime}\right)$ in $\mathcal{H} A L G^{\prime}$ are such that $h_{i}$ is the restriction of $h_{j}$ for all $(i, j) \in$ $R_{\leq}$. The quantification space $\mathcal{D}^{\mathcal{H} A L G^{\prime}}$ consists of the signature extensions with $A L G$ variables and nominal symbols variables. We denote $[\lambda]$ by $\square$ and $\langle\lambda\rangle$ by $\diamond$. Since $\Lambda$ is fixed we may denote the signatures of $\mathcal{H} A L G^{\prime}$ simply by $(\Sigma$, Nom), with $\Sigma$ being an $A L G$-signature.

The argument that the amalgamation of constrained models yields a constraint model, which represents the reflection condition of Thm. 3.1, is a bit more complex than in the previous examples. It is an immediate consequence of the fact that, in the case of a pushout square of $A L G$ signatures like below

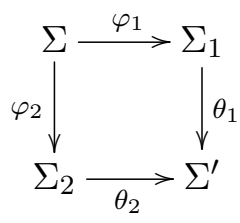

the amalgamation of inclusive algebra homomorphisms yields an inclusive algebra homomorphism. Thus let $h_{k}: A_{k} \rightarrow B_{k}$ be inclusive $\Sigma_{k}$-algebra homomorphisms for $k \in\{1,2\}$. Let $\left(h_{1} \otimes h_{2}\right): A_{1} \otimes A_{2} \rightarrow$ $B_{1} \otimes B_{2}$ be their (unique) amalgamation (here we apply the amalgamation property for homomorphisms, a property enjoyed by $A L G$ according to the literature, e.g. [14]). Since this is a homomorphism it suffices to show that for each sort $s,\left(h_{1} \otimes h_{2}\right)_{s}$ is an inclusion. By the pushout construction in the category of $A L G$ signatures there exists $k \in\{1,2\}$ and $s_{k}$ sort in $\Sigma_{k}$ such that $s=\theta_{k}\left(s_{k}\right)$. Then $\left(h_{1} \otimes h_{2}\right)_{s}=\left(h_{k}\right)_{s_{k}}$, hence $\left(h_{1} \otimes h_{2}\right)_{s}$ is inclusion since $\left(h_{k}\right)_{s_{k}}$ is inclusion by hypothesis.

Note that like in $\mathcal{H} F O L, \mathcal{H} R E L^{\prime}, \mathcal{H} P A^{\prime}$ we do not need to distinguish between the Boolean connectives at the level of $A L G$ and at the level of $\mathcal{H} A L G^{\prime}$, however unlike in the above mentioned cases we do need to distinguish between quantifiers at the level of $A L G$ and at the level of $\mathcal{H} A L G^{\prime}$. For example if we consider $\Sigma$ consisting of one constant $a$ only, Nom $=\emptyset$ and the $(\Sigma, \operatorname{Nom})$-model $(M, R)$ such that $|R|=\{i, j\}, R_{\leq}\{(i, j)\}, M_{i}$ a singleton, and $M_{j}$ having two elements. Then $(M, R) \not \nvdash^{j}(\forall x) x=a$ but $(M, R) \models^{j}(\emptyset x) x=a$.

\section{Preservation along model homomorphisms}

This short section is devoted to preservation of (satisfaction of) senstences along model homomorphisms in hybridized institutions, and it has only a technical significance.

Definition 4.1 (Preservation along model homomorphisms). In an institution $\mathcal{I}$, a sentence $\rho$ is preserved along a model homomorphism $h: M \rightarrow M^{\prime}$ if and only if $M \models \rho$ implies $M^{\prime} \models \rho$.

Example 4.1. The following preservation results constitute simple exercises and moreover most of them are also known from the literature (e.g. [14]).

- For any $P L$ signature $P$, each $\pi \in P$ is preserved by all model homomorphisms.

- In $R E L$ the relational atoms $\pi\left(t_{1}, \ldots, t_{n}\right)$ and the equational atoms $t=t^{\prime}$ are preserved by all model homomorphisms.

- In $R E L$ the negations of equational atoms $t \neq t^{\prime}$ are preserved by the injective model homomorphisms. 
- In REL a model homomorphism $h: M \rightarrow M^{\prime}$ is closed when $M_{\pi}=h^{-1}\left(M_{\pi}^{\prime}\right)$ for each relational symbol $\pi$ of the respective signature. Then in $R E L$ the negations atoms $\neg \pi\left(t_{1}, \ldots, t_{n}\right)$ are preserved by all closed model homomorphisms.

- In $A L G$ the equations $t=t^{\prime}$ are preserved by all model homomorphisms and the negations of equations $t \neq t^{\prime}$ are preserved by all injective homomorphisms.

- In $P A$ the existence equations $t \stackrel{e}{=} t^{\prime}$ are preserved by all model homomorphisms.

- In $P A$ a model homomorphism $h: M \rightarrow M^{\prime}$ is closed when for each partial operation symbol $\sigma \in P F_{w \rightarrow s}$ of the respective signature and for each $m \in M_{w}, M_{\sigma}^{\prime}\left(h_{w}(m)\right)$ is defined implies that $M_{\sigma}(m)$ is also defined. Then in $P A$ the negations of existence equations $t \stackrel{e}{=} t^{\prime}$ are preserved by all injective and closed model homomorphisms.

- In $P L, R E L, A L G$ and $P A$ the sentences preserved by a model homomorphism $h$ are closed under conjunction $\wedge$ and disjunction $\vee$.

- In $P L, R E L, A L G$ and $P A$ if a sentence $\rho$ is preserved by all $\chi$-expansions of $h$ then $(\exists \chi) \rho$ is preserved by $h$. Consequently the sentences preserved by all model homomorphisms are closed under existential quantifications.

- The universally quantified atoms $(\forall X) \pi\left(t_{1}, \ldots, t_{n}\right)$ (in $R E L$ ), $(\forall X) t=t^{\prime}$ (in $A L G, R E L$ ), and $(\forall X) t \stackrel{e}{=} t^{\prime}$ (in $P A$ ) are preserved by the surjective homomorphisms.

Definition 4.2 (Local preservation along model homomorphisms). In a hybridization of an institution $\mathcal{I}$, a sentence $\rho$ is locally preserved along a model homomorphism $h:(M, R) \rightarrow\left(M^{\prime}, R^{\prime}\right)$ if and only if for each $s \in|R|,(M, R) \models^{s} \rho$ implies $\left(M^{\prime}, R^{\prime}\right) \mid=^{h(s)} \rho$.

Definition 4.3. For any signature morphism $\varphi: \Sigma \rightarrow \Sigma^{\prime}$ in an institution and for any $\Sigma$-model homomorphism $h: M \rightarrow N$, we say that $\varphi$ lifts forward (backwards) $h$ when for each $\varphi$-expansion $M^{\prime}\left(N^{\prime}\right)$ of $M$ $(N)$ there exists a $\varphi$-expansion $h^{\prime}: M^{\prime} \rightarrow N^{\prime}$ of $h$.

\section{Proposition 4.1.}

1. Each nominal is locally preserved along any homomorphism.

2. For any $\rho \in \operatorname{Sen}^{\mathcal{I}}(\Sigma)$ and any $(\Sigma$, Nom, $\Lambda)$-homomorphism $h:(M, R) \rightarrow\left(M^{\prime}, R^{\prime}\right)$, if $\rho$ is preserved along each $h_{i}, i \in|R|$, then $\rho$ is locally preserved along $h$.

3. If $\rho_{1}$ and $\rho_{2}$ are locally preserved along $h$ then $\rho_{1} \wedge \rho_{2}$ and $\rho_{1} \vee \rho_{2}$ are also locally preserved along $h$.

4. If $\rho$ is locally preserved along $h$ then $@_{i} \rho$ is also locally preserved along $h$.

5. If $\rho$ is locally preserved along $h:(M, R) \rightarrow\left(M^{\prime}, R^{\prime}\right)$ and $h$ has the property that for each $\left(h(s), s^{\prime}\right) \in$ $R_{\lambda}^{\prime}$ there exists $s^{\prime \prime} \in h_{s t}^{-1}\left(s^{\prime}\right)$ such that $\left(s, s^{\prime \prime}\right) \in R_{\lambda}$ then $[\lambda](\rho)$ is also locally preserved along $h$.

6. If $\rho$ is locally preserved along $h$ then $\langle\lambda\rangle(\rho)$ is also locally preserved along $h$.

7. If $\rho$ is locally preserved along all $\chi$-expansions of $h:(M, R) \rightarrow\left(M^{\prime}, R^{\prime}\right)$ and $\chi$ lifts forward (backwards) $h$ then $(\exists \chi) \rho((\forall \chi) \rho)$ is locally preserved along $h$.

Proof. 1. Let $i \in$ Nom. Let us assume $(M, R) \models^{s} i$, which means $s=R_{i}$. Since $h\left(R_{i}\right)=R_{i}^{\prime}$ it follows that $h(s)=R_{i}^{\prime}$ which means $\left(M^{\prime}, R^{\prime}\right) \models{ }^{h(s)} i$.

2. Let $s \in|R|$ and let $(M, R) \models^{s} \rho$. Since $\rho \in \operatorname{Sen}^{\mathcal{I}}(\Sigma)$ it follows that $M_{s} \models^{\mathcal{I}} \rho$. Since $h_{s}: M_{s} \rightarrow$ $M_{h(s)}^{\prime}$ preserves $\rho$ it follows that $M_{h(s)}^{\prime} \models^{\mathcal{I}} \rho$, which means $\left(M^{\prime}, R^{\prime}\right) \models^{h(s)} \rho$. 
3. Assume $(M, R) \models{ }^{s} \rho_{1} \wedge \rho_{2}$ which means $(M, R) \models^{s} \rho_{i}$ for each $i \in\{1,2\}$. Then by hypothesis $\left(M^{\prime}, R^{\prime}\right) \models^{h(s)} \rho_{i}$ for each $i \in\{1,2\}$ hence $\left(M^{\prime}, R^{\prime}\right) \models{ }^{h(s)} \rho_{1} \wedge \rho_{2}$.

If $(M, R) \models^{s} \rho_{1} \vee \rho_{2}$ then there exists $i \in\{1,2\}$ such that $(M, R) \models^{s} \rho_{i}$. By hypothesis it follows that $\left(M^{\prime}, R^{\prime}\right) \models{ }^{h(s)} \rho_{i}$ hence $\left(M^{\prime}, R^{\prime}\right) \models{ }^{h(s)} \rho_{1} \vee \rho_{2}$.

4. Assume $(M, R) \models \models^{s} @_{i} \rho$. This means $(M, R) \models{ }^{R_{i}} \rho$. By hypothesis $\left(M^{\prime}, R^{\prime}\right) \models{ }^{h\left(R_{i}\right)} \rho$ which means $\left(M^{\prime}, R^{\prime}\right) \models{ }^{R_{i}^{\prime}} \rho$. Hence $\left(M^{\prime}, R^{\prime}\right) \models=^{h(s)} @_{i} \rho$.

5. Assume $(M, R) \models^{s}[\lambda]\left(\rho_{1}, \ldots, \rho_{n}\right)$. Let any $\left(s_{1}^{\prime}, \ldots, s_{n}^{\prime}\right)$ such that $\left(h(s), s_{1}^{\prime}, \ldots, s_{n}^{\prime}\right) \in R_{\lambda}^{\prime}$. By hypothesis there exists $s_{1} \in h^{-1}\left(s_{1}^{\prime}\right), \ldots, s_{n} \in h^{-1}\left(s_{n}^{\prime}\right)$ such that $\left(s, s_{1}, \ldots, s_{n}\right) \in R_{\lambda}$. By our assumption we have that there exists $1 \leq k \leq n$ such that $(M, R) \models{ }^{s_{k}} \rho_{k}$. By hypothesis it follows that $\left(M^{\prime}, R^{\prime}\right) \models{ }^{h\left(s_{k}\right)} \rho_{k}$ which means $\left(M^{\prime}, R^{\prime}\right) \models{ }^{\prime}{ }_{k}^{\prime} \rho_{k}$.

6. Assume $(M, R) \models{ }^{s}\langle\lambda\rangle(\rho)$, which means $(M, R) \models=^{s^{\prime}} \rho$ for some $\left(s, s^{\prime}\right) \in R_{\lambda}$. By hypothesis $\left(M^{\prime}, R^{\prime}\right) \models^{h\left(s^{\prime}\right)} \rho$ hence $\left(M^{\prime}, R^{\prime}\right) \models{ }^{h(s)}\langle\lambda\rangle(\rho)$.

7. Assume $(M, R) \models^{s}(\exists \chi) \rho$. Then there exists a $\chi$-expansion $(\bar{M}, \bar{R})$ of $(M, R)$ such that $(\bar{M}, \bar{R}) \models^{s}$ $\rho$. By the lifting condition there exists a $\chi$-expansion $h^{\prime}:(\bar{M}, \bar{R}) \rightarrow\left(\overline{M^{\prime}}, \overline{R^{\prime}}\right)$ of $h$. By the hypotheses we have that $\left(\overline{M^{\prime}}, \overline{R^{\prime}}\right) \models{ }^{h^{\prime}(s)=h(s)} \rho$. This shows $\left(M^{\prime}, R^{\prime}\right) \models{ }^{h(s)}(\exists \chi) \rho$.

Assume $(M, R) \models^{s}(\forall \chi) \rho$. Let $\left(\overline{M^{\prime}}, \overline{R^{\prime}}\right)$ be any $\chi$-expansion of $\left(M^{\prime}, R^{\prime}\right)$. By the lifting condition there exists a $\chi$-expansion $h^{\prime}:(\bar{M}, \bar{R}) \rightarrow\left(\overline{M^{\prime}}, \overline{R^{\prime}}\right)$ of $h$. It follows that $(\bar{M}, \bar{R}) \models^{s} \rho$. By the hypothesis that $\rho$ is locally preserved by $h^{\prime}$ it follows that $\left(\overline{M^{\prime}}, \overline{R^{\prime}}\right) \models{ }^{h(s)=h^{\prime}(s)} \rho$. This shows $\left(M^{\prime}, R^{\prime}\right) \models{ }^{h(s)}(\forall \chi) \rho$.

Remark 4.1. In the examples the conditions on forward (backwards) lifting of Prop. 4.1 may be obtained by the following general methods. We assume a given $(\Sigma$, Nom, $\Lambda)$-homomorphism $h:(M, R) \rightarrow\left(M^{\prime}, R^{\prime}\right)$.

1. If $\left.\chi\right|_{\mathcal{I}}$ lifts forward each $h_{i}, i \in|R|$, and $\left.\chi\right|_{R E L}$ is injective, then $\chi$ lifts forward $h$. Indeed, for any

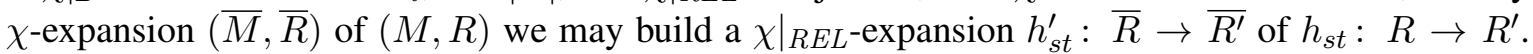
Also for each $i \in|R|=|\bar{R}|$ there exists a $\left.\chi\right|_{\mathcal{I}^{-}}$-expansion $h_{i}^{\prime}: \bar{M}_{i} \rightarrow{\overline{M^{\prime}}}_{h_{s t}(i)}$ of $h_{i}$. These define a $\chi$-expansion $h^{\prime}:(\bar{M}, \bar{R}) \rightarrow\left(\overline{M^{\prime}}, \overline{R^{\prime}}\right)$ of $h$.

2. If $\left.\chi\right|_{\mathcal{I}}$ lifts backwards each $h_{i}, i \in|R|,\left.\chi\right|_{R E L}$ is injective, and $h_{s t}$ is surjective, then $\chi$ lifts backwards $h$. Indeed, for any $\chi$-expansion $\left(\overline{M^{\prime}}, \overline{R^{\prime}}\right)$ of $\left(M^{\prime}, R^{\prime}\right)$, we may build a $\left.\chi\right|_{R E L}$-expansion $h_{s t}^{\prime}: \bar{R} \rightarrow \overline{R^{\prime}}$

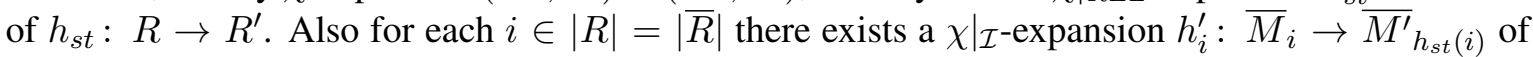
$h_{i}$. These define a $\chi$-expansion $h^{\prime}:(\bar{M}, \bar{R}) \rightarrow\left(\overline{M^{\prime}}, \overline{R^{\prime}}\right)$ of $h$.

However, in the applications of this methods, in both above cases we have to choose the expansions in such way that when starting with a Mod'-homomorphism $h$ we end up with the expansions $h^{\prime}$ also being Mod'-homomorphism.

Example 4.2. The last three items of Ex. 4.1 may be derived from the previous items of Ex. 4.1 via the result of Prop. 4.1 by considering $|R|$ and $\left|R^{\prime}\right|$ singleton sets.

Example 4.3. In $\mathcal{H} R E L^{\prime}$ we fix a signature and consider a variable $x$ for the corresponding $R E L$-signature, a nominal variable $n$, and a modality variable $\lambda$. Let $\pi$ be a unary relation symbol in the signature. Let us show that $(\exists x, n, \lambda) @_{n}(\pi(x) \Rightarrow\langle\lambda\rangle n)$ is locally preserved along any homomorphism $h:(M, R) \rightarrow$ $\left(M^{\prime}, R^{\prime}\right)$ such that each $h_{i}, i \in|R|$, is closed.

For this we replace $\Rightarrow$ by its equivalent in terms of $\neg$ and $\vee$ and we apply Prop. 4.1 recursively on the structure of the sentence, with the base case following from Ex. 4.1, namely that $\neg \pi(x)$ is preserved along closed homomorphisms in $R E L$. The last recursion step, related to the quantifier, requires more attention. For this we use the corresponding part of Remark 4.1. Note that the quantification corresponds to 
an inclusive signature morphism that just adds symbols to the original signature. We need to pay attention to the fact that $\left(\overline{M^{\prime}}, \overline{R^{\prime}}\right)$ should be a Mod'-model, which means that for any $j, k \in\left|R^{\prime}\right|$ we have

1. $\left|\bar{M}_{j}{ }_{j}\right|=\left|\overline{M^{\prime}}{ }_{k}\right|$, i.e. the possible worlds of $\overline{M^{\prime}}$ share the same underlying universes, and

2. for each constant $c,\left({\overline{M^{\prime}}}_{j}\right)_{c}=\left(\overline{M^{\prime}}{ }_{k}\right)_{c}$, i.e. the possible worlds of $\overline{M^{\prime}}$ share the same interpretations of the constants.

The former point follows from the fact that the expansions keep the underlying universes unchanged, i.e. $\left|\overline{M^{\prime}}{ }_{j}\right|=\left|M_{j}^{\prime}\right|$ and $\left|\overline{M^{\prime}}{ }_{k}\right|=\left|M_{k}^{\prime}\right|$, and because $\left|M_{j}^{\prime}\right|=\left|M_{k}^{\prime}\right|$ since $\left(M^{\prime}, R^{\prime}\right)$ is a $\operatorname{Mod}^{\prime}$-model. In order to fulfill the latter point we have to define for each $j \in\left|R^{\prime}\right|, i \in|R|,\left(\bar{M}_{j}^{\prime}\right)_{x}=h_{i}\left(\left(\bar{M}_{h_{s t}(i)}\right)_{x}\right)$ (the correctness of this definition follows by the fact that $(\bar{M}, \bar{R})$ is a Mod'-model). In the case of the constants $c$ of the original signature we use that fact that $\left(M^{\prime}, R^{\prime}\right)$ is a Mod'-model in order to get the following equalities: $\left(\bar{M}_{j}^{\prime}\right)_{c}=\left(M_{j}^{\prime}\right)_{c}=\left(M_{k}^{\prime}\right)_{c}=\left(\bar{M}_{k}^{\prime}\right)_{c}$.

Now let us show that $(\forall x, n, \lambda) @_{n}(\pi(x) \Rightarrow\langle\lambda\rangle n)$ is locally preserved along any homomorphism $h:(M, R) \rightarrow$ $\left(M^{\prime}, R^{\prime}\right)$ such that $h_{s t}$ is surjective and each $h_{i}, i \in|R|$, is closed and surjective. Apart of the quantifier step the argument for this case shares with the argument for the existential variant above. For the quantifier step we use the backwards lifting part of Remark 4.1. We make sure that the expansion $(\bar{M}, \bar{R})$ is a $\operatorname{Mod}^{\prime}$-model as follows:

1. for any $i, j \in|R|$, by the basic model reduct/expansion properties and because $(M, R)$ is a $\operatorname{Mod}^{\prime}$ model we have that $\left|\bar{M}_{i}\right|=\left|M_{i}\right|=\left|M_{j}\right|=\left|\bar{M}_{j}\right|$ and that $\left(\bar{M}_{i}\right)_{c}=\left(M_{i}\right)_{c}=\left(M_{j}\right)_{c}=\left(\bar{M}_{j}\right)_{c}$ for any constant $c$ of the original signature, and

2. since $\left(\overline{M^{\prime}}, \overline{R^{\prime}}\right)$ is a $\operatorname{Mod}^{\prime}$-model we have that for any $i, j \in|R|, h_{i}^{-1}\left(\left(\overline{M^{\prime}}{ }_{i}\right)_{x}\right)=h_{j}^{-1}\left(\left(\overline{M^{\prime}}{ }_{j}\right)_{x}\right)$; therefore we pick $a \in h_{i}^{-1}\left(\left(\overline{M^{\prime}} i\right)_{x}\right)$ and for each $i \in|R|$ we define $\left(\bar{M}_{i}\right)_{x}=a$.

\section{Inclusion systems for models of hybridized institutions}

The goal of this section is to establish the concept of sub-model for the models of hybridized institutions and to develop results about preservation by sub-models. Any proper concept of sub-model comes as part of a so-called 'factorization system'; in our case we use the brand of factorization systems known as 'inclusion systems'. The structure of the section is as follows:

1. We review briefly the concepts from the theory of inclusion systems that are necessary for our work here.

2. We develop inclusion systems for the categories of models of hybridized institutions; this yields the concept of sub-model in hybridized institutions.

3. We develop preservation results by sub-models in hybridized institutions.

\subsection{Inclusion systems}

Inclusion systems were introduced in [18] as a categorical device supporting an abstract general study of structuring of specification and programming modules that is independent of any underlying logic. They have been used in a series of general module algebra studies such as $[14,17,18,22]$ but also for developing axiomatizability [13, 14, 33] and definability [1] results within the framework of the so-called 'institution-independent model theory' [14]. Inclusion systems capture categorically the concept of settheoretic inclusion in a way reminiscent of how the rather notorious concept of factorization system [8] captures categorically the set-theoretic injections; however in many applications the former are more convenient than the latter. Here we first recall from the literature the basics of the theory of inclusion systems that are necessary for the developments of this paper. 
The definition below can be found in the recent literature on inclusion systems (e.g. [14]) and differs slightly from the original one of [18].

Definition 5.1 (Inclusion systems). $\langle I, \mathcal{E}\rangle$ is a inclusion system for a category $\mathbb{C}$ if $I$ and $\mathcal{E}$ are two subcategories with $|I|=|\mathcal{E}|=|\mathbb{C}|$ such that

1. I is a partial order (with the ordering relation denoted by $\subseteq$ ), and

2. every arrow $f$ in $\mathbb{C}$ can be factored uniquely as $f=e_{f} ; i_{f}$ with $e_{f} \in \mathcal{E}$ and $i_{f} \in I$.

The arrows of $I$ are called abstract inclusions, and the arrows of $\mathcal{E}$ are called abstract surjections. The domain of the inclusion $i_{f}$ in the factorization of $f$ is called the image of $f$ and is denoted as $\operatorname{Im}(f)$ or $f(A)$ when $A$ is a domain of $f$. An inclusion $i: A \rightarrow B$ may be also denoted simply by $A \subseteq B$ and we may say that $A$ is a sub-object of $B$.

The inclusion system

- is epic when all abstract surjections are epis, and

- is co-well-powered when for each $A \in|\mathbb{C}|$ the quotient class $q(A)=\{e: A \rightarrow B \mid e \in \mathcal{E}\} / \cong_{A}$ is a set, where $\cong_{A}$ is the equivalence defined by $e \cong_{A} e^{\prime}$ if and only if there exists an isomorphism $i \in \mathbb{C}$ such that $e ; i=e^{\prime}$.

In [12] it is shown that the class $I$ of the abstract inclusions determines the class $\mathcal{E}$ of the abstract surjections. In this sense, [12] gives an explicit equivalent definition of inclusion systems which uses only the class $I$ of the abstract inclusions.

Example 5.1. The standard example of inclusion system is that from Set, with set theoretic inclusions in the role of the abstract inclusions and the surjective functions in the role of the abstract surjections. It is easy to note that this is epic and co-well-powered.

Example 5.2. A $\Sigma$-model homomorphism $h: M \rightarrow M^{\prime}$ in $R E L$

- is closed when for each relation symbol $\pi$ in $\Sigma, M_{\pi}=h^{-1}\left(M_{\pi}^{\prime}\right)$, and

- is strong when for each relation symbol $\pi$ in $\Sigma, h\left(M_{\pi}\right)=M_{\pi}^{\prime}$.

A $(S, T F, P F)$-model homomorphism $h: M \rightarrow M^{\prime}$ in $P A$

- is closed when for each $\sigma \in P F_{w \rightarrow s}$ and each $m \in M_{w}, M_{\sigma}(m)$ is defined whenever $M_{\sigma}^{\prime}\left(h_{w}(m)\right)$ is defined, and

- is full when for each partial operation symbol $\sigma \in P F_{w \rightarrow s}$ and each $m \in M_{w}$, if $M_{\sigma}^{\prime}\left(h_{w}(m)\right)$ is defined then there exists $n \in M_{w}$ such that $M_{\sigma}(n)$ is defined and $h_{w}(m)=h_{w}(n)$.

The inclusion systems listed below can be found in the model theory literature (e.g. [14]).

\begin{tabular}{c|l|l|l} 
INSTITUTION & INCLUSION SYSTEM & $\mathcal{E}$ & $I$ \\
\hline$P L$ & $\begin{array}{c}\text { closed } \\
\text { strong }\end{array}$ & $\begin{array}{l}\text { all homomorphisms } \\
\text { identities }\end{array}$ & $\begin{array}{l}\text { identities } \\
\text { all homomorphisms }\end{array}$ \\
\hline$R E L$ & $\begin{array}{l}\text { closed } \\
\text { strong }\end{array}$ & $\begin{array}{l}\text { surjective homomorphisms } \\
\text { strong surjective homomorphisms }\end{array}$ & $\begin{array}{l}\text { closed sub-models } \\
\text { plain sub-models }\end{array}$ \\
\hline$A L G$ & standard & surjective homomorphisms & sub-algebras \\
\hline$P A$ & closed & $\begin{array}{l}\text { epi homomorphisms } \\
\text { surjective homomorphisms } \\
\text { full surjective homomorphisms }\end{array}$ & $\begin{array}{l}\text { closed sub-algebras } \\
\text { full sub-algebras } \\
\text { plain sub-algebras }\end{array}$ \\
& full & strong & 19
\end{tabular}


It is easy to note that all these inclusion systems are epic and co-well-powered.

Definition 5.2 (Quasi-variety). A sub-class of objects $\mathcal{Q} \subseteq|\mathbb{C}|$ of a category $\mathbb{C}$ with a designated inclusion system

- is closed under sub-models when for each $A \subseteq B, B \in \mathcal{Q}$ implies $A \in \mathcal{Q}$,

- is closed under small products when for any set $I$ and objects $A_{i} \in Q$, for $i \in I$, we have that $\prod_{i \in I} A_{i} \in Q$, and

- is quasi-variety (in $\mathbb{C}$ ) when it is both closed under sub-models and under small products.

Fact 5.1. Any intersection of quasi-varieties is a a quasi-variety.

\subsection{Inclusion systems for models in hybridized institutions}

The following theorem provides a set of sufficient conditions for categories of models of hybridized institutions to posses a proper inclusion system in the sense of having the necessary properties required for developing initial semantics by means of quasi-varieties. These conditions have a rather technical nature and are given with the intention to have them checked rather easily in the concrete situations.

Theorem 5.1. Given a base institution $\mathcal{I}$, a hybridization $\left(\operatorname{Sign}^{\mathcal{H I}}, \operatorname{Sen}^{\mathcal{H I}}, \operatorname{Mod}^{\prime}, \models\right)$ of $\mathcal{I}$, a $\mathcal{H} \mathcal{I}$-signature $(\Sigma, \mathrm{Nom}, \Lambda)$, an inclusion system for the category of the (Nom, $\Lambda)$-models, and an inclusion system $\left(I^{\Sigma}, \mathcal{E}^{\Sigma}\right)$ for the category of $\Sigma$-models, we assume the following conditions:

1. For each $\operatorname{Mod}^{\prime}(\Sigma, \operatorname{Nom}, \Lambda)$-homomorphism $h:(M, R) \rightarrow\left(M^{\prime}, R^{\prime}\right)$ let us factor each $h_{i}, i \in|R|$, through $\left(I^{\Sigma}, \mathcal{E}^{\Sigma}\right)$ as follows:

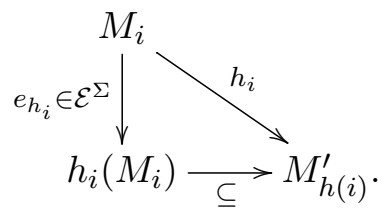

Let $h(M):|R| \rightarrow|\operatorname{Mod}(\Sigma)|$ be defined by $h(M)_{i}=h_{i}\left(M_{i}\right)$. Then $\left(\left\{e_{h_{i}}|i \in| R \mid\right\}, 1_{R}\right)$ constitutes $a \operatorname{Mod}^{\prime}$-homomorphism $(M, R) \rightarrow(h(M), R)$.

2. For any $\operatorname{Mod}^{\mathcal{H I}}(\Sigma, \mathrm{Nom}, \Lambda)$-homomorphism $h:(M, R) \rightarrow\left(M^{\prime}, R^{\prime}\right)$ such that $(M, R),\left(M^{\prime}, R^{\prime}\right) \in$ $\mid \operatorname{Mod}^{\prime}(\Sigma$, Nom, $\Lambda) \mid$ and for each $i \in|R|, h_{i}=\left(M_{i} \subseteq M_{i}^{\prime}\right)$ we have that $h$ is a Mod'-homomorphism. The situation when in addition $h_{\text {st }}$ is abstract inclusion of $(\mathrm{Nom}, \Lambda)$-models is denoted by $(M, R) \subseteq$ $\left(M^{\prime}, R^{\prime}\right)$.

3. For each $\operatorname{Mod}^{\prime}(\Sigma, \operatorname{Nom}, \Lambda)$-homomorphism $h:(M, R) \rightarrow\left(M^{\prime}, R^{\prime}\right)$ let us factor $h_{\text {st }}$ through the inclusion system of (Nom, $\Lambda)$-models as follows:

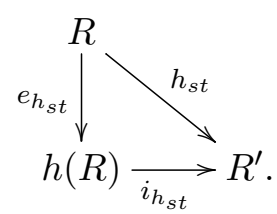

Then there exists $\left(\cup^{e_{h}}\right.$ st $\left.M, h(R)\right) \in\left|\operatorname{Mod}^{\prime}(\Sigma, N o m, \Lambda)\right|$ such that

$-(M, R) \subseteq\left(e_{h_{s t}} ; \cup^{e_{s t}} M, R\right)$, and 
- the following universal property holds: for each Mod'-homomorphism $\left(f, e_{h_{s t}}\right):(M, R) \rightarrow$ $\left(N^{\prime}, h(R)\right)$ there exists an unique $\operatorname{Mod}^{\prime}$-homomorphism $\left(f^{\prime}, 1_{h(R)}\right):\left(\cup^{e^{h}}{ }_{s t} M, h(R)\right) \rightarrow\left(N^{\prime}, h(R)\right)$ such that the diagram below commutes
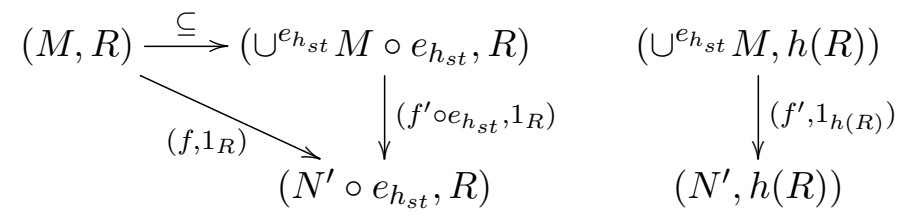

and moreover, when $\left\{f_{i}|i \in| R \mid\right\}$ are inclusions, then $\left\{f_{j}^{\prime}|j \in| h(R) \mid\right\}$ are inclusions too.

Then $\operatorname{Mod}^{\prime}(\Sigma, \operatorname{Nom}, \Lambda)$ admits an inclusion system as follows:

- the abstract inclusions are the homomorphisms $(M, R) \subseteq\left(M^{\prime}, R^{\prime}\right)$, and

- the abstract surjections are the homomorphisms $h:(M, R) \rightarrow\left(M^{\prime}, R^{\prime}\right)$ such that $h_{s t}: R \rightarrow R^{\prime}$ is abstract surjection (of (Nom, $\Lambda$ )-models) and $M^{\prime}=\cup^{h s t} h(M)$.

Proof. For any $h:(M, R) \rightarrow\left(M^{\prime}, R^{\prime}\right)$ let $\cup^{h} M$ abbreviate $\cup^{h s t} M$.

We first prove that if $h:(M, R) \rightarrow\left(M^{\prime}, R^{\prime}\right)$ and $h^{\prime}:\left(M^{\prime}, R^{\prime}\right) \rightarrow\left(M^{\prime \prime}, R^{\prime \prime}\right)$ are abstract surjections then their composition $h ; h^{\prime}$ is an abstract surjection too. Since $h_{s t}$ and $h_{s t}^{\prime}$ are abstract surjections of (Nom, $\Lambda$ )-models it follows immediately that $h_{s t} ; h_{s t}^{\prime}$ is abstract surjection of (Nom, $\Lambda$ )-models too. It remains to show that $M^{\prime \prime}=\cup^{h ; h^{\prime}}\left(h ; h^{\prime}\right)(M)$.

In the commutative diagram below, for each $i \in|R|$ and for each $j \in\left|R^{\prime}\right|$ we let $\left(e_{h}\right)_{i},\left(e_{1}\right)_{i},\left(e_{h^{\prime}}\right)_{j} \in$ $\mathcal{E}^{\Sigma}$, and $\left(i_{h}\right)_{i},\left(i_{1}\right)_{i},\left(i_{h^{\prime}}\right)_{j} \in I^{\Sigma}$ (where each $\left(e_{1}\right)_{i} ;\left(i_{1}\right)_{i}$ is the factorization of $\left.\left(i_{h}\right)_{i} ;\left(e_{h^{\prime}}\right)_{h(i)}\right)$.

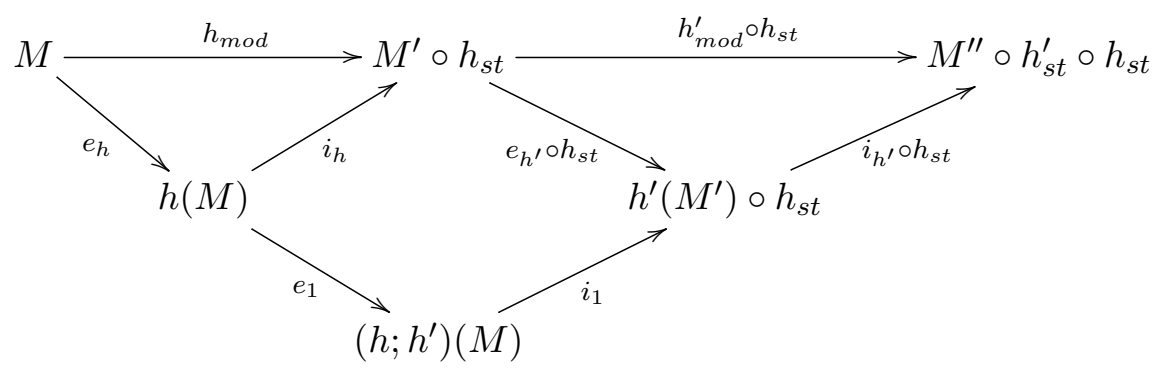

Since $e_{h} ; e_{1}$ consists of abstract inclusions and $i_{1} ;\left(i_{h^{\prime}} \circ h_{s t}\right)$ consists of abstract inclusions, by the uniqueness property of factorizations it follows that the codomain of $e_{1}$ is $\left(h ; h^{\prime}\right)(M)$.

For any $\left(g, 1_{R}\right):(N, R) \rightarrow\left(N^{\prime}, R\right)$ and any $h_{s t}: R \rightarrow R^{\prime}$ abstract surjection of (Nom, $\left.\Lambda\right)$-models, by the universal property of $\left(\cup^{h} N, R^{\prime}\right)$ we define $\cup^{h} g: \cup^{h} N \rightarrow \cup^{h} N^{\prime}$ such that the diagram below commutes:

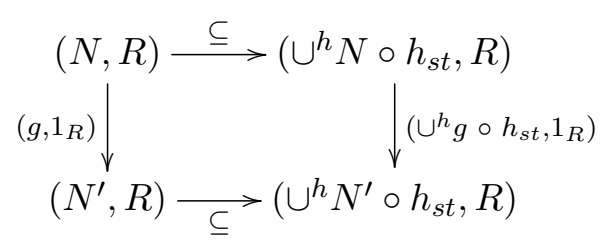


For each $\left(N, R^{\prime}\right)$, by the universal property of $\left(\cup^{h}\left(N \circ h_{s t}\right), R^{\prime}\right)$ we define $\epsilon_{N}: \cup^{h}\left(N \circ h_{s t}\right) \rightarrow N$ such that the diagram below commutes:

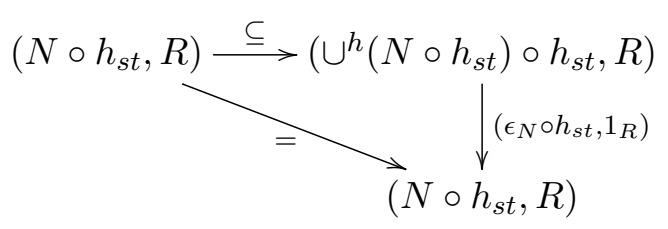

By the universal properties of $\left(\cup^{h} h(M), R^{\prime}\right)$ and of $\left(\cup^{h}\left(M^{\prime} \circ h_{s t}\right), R^{\prime}\right)$ we obtain that both the inner squares of the diagram below commute, hence the outer square commutes too.

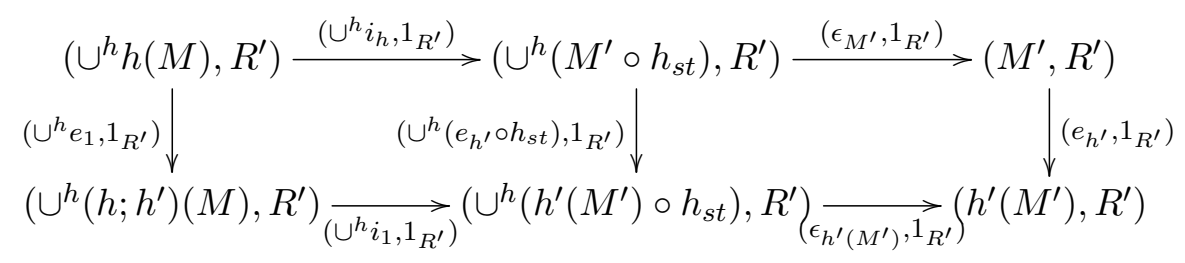

Since by the hypotheses of the theorem $\epsilon_{M^{\prime}}$ consists of inclusions and since $\left(M^{\prime}, R^{\prime}\right)=\left(\cup^{h} h(M), R^{\prime}\right)$ (because $h$ is abstract surjection) we have that $\cup^{h} i_{h} ; \epsilon_{M^{\prime}}$ is an identity, hence $\cup^{h} e_{1} ;\left(\cup^{h} i_{1} ; \epsilon_{h^{\prime}\left(M^{\prime}\right)}\right)=e_{h^{\prime}}$. Since for each $j \in\left|R^{\prime}\right|$ we have that $\left(e_{h^{\prime}}\right)_{j}$ is an abstract surjection, by the uniqueness property of the factorization we get that $\cup^{h} i_{1} ; \epsilon_{h^{\prime}\left(M^{\prime}\right)}$ is an identity, hence

$$
\left(\cup^{h}\left(h ; h^{\prime}\right)(M), R^{\prime}\right)=\left(h^{\prime}\left(M^{\prime}\right), R^{\prime}\right) .
$$

If we showed that for each $(N, R)$ we have that

$$
\left(\cup^{h ; h^{\prime}} N, R^{\prime \prime}\right)=\left(\cup^{h^{\prime}} \cup^{h} N, R^{\prime \prime}\right)
$$

then by considering $\left(\left(h ; h^{\prime}\right)(M), R\right)$ in the role of $(N, R)$, from $(4)$ and from the fact that $\left(M^{\prime \prime}, R^{\prime \prime}\right)=$ $\left(\cup^{h^{\prime}} h^{\prime}\left(M^{\prime}\right), R^{\prime \prime}\right)$ (since $h^{\prime}$ is abstract surjection) it follows that $\left(M^{\prime \prime}, R^{\prime \prime}\right)=\left(\cup^{h ; h^{\prime}}\left(h ; h^{\prime}\right)(M), R^{\prime \prime}\right)$. Modulo (5), this completes the proof that the abstract surjections are closed under composition.

We now prove the relation (5) by the uniqueness of $\left(\cup^{h ; h^{\prime}} N, R^{\prime \prime}\right)$ (which follows easily from its universal property) and by establishing that $\left(\cup^{h^{\prime}} \cup^{h} N, R^{\prime \prime}\right)$ fulfills the universal property of $\left(\cup^{h ; h^{\prime}} N, R^{\prime \prime}\right)$. For any $\left(f, 1_{R}\right):(N, R) \rightarrow\left(N^{\prime \prime} \circ h_{s t}^{\prime} \circ h_{s t}, R\right)$ by the universal property of $\left(\cup^{h} N, R^{\prime}\right)$ we get $\left(f^{\prime}, 1_{R^{\prime}}\right):\left(\cup^{h} N, R^{\prime}\right) \rightarrow$ $\left(N^{\prime \prime} \circ h_{s t}^{\prime}, R^{\prime}\right)$ and by the universal property of $\left(\cup^{h^{\prime}} \cup^{h} N, R^{\prime \prime}\right)$ we further get $\left(f^{\prime \prime}, 1_{R^{\prime \prime}}\right):\left(\cup^{h^{\prime}} \cup^{h} N, R^{\prime \prime}\right) \rightarrow$ $\left(N^{\prime \prime}, R^{\prime \prime}\right)$. The uniqueness of $f^{\prime \prime}$ propagates through the uniqueness of $f^{\prime}$. Moreover when $f$ consists of inclusions then $f^{\prime}$ consists of inclusions too which implies that $f^{\prime \prime}$ also consists of inclusions.

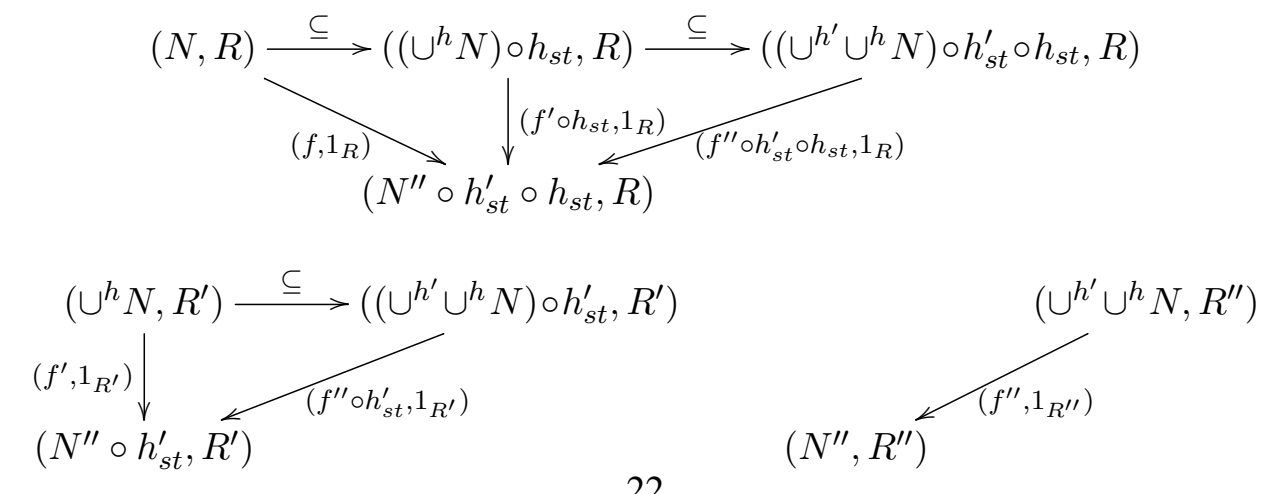


Any homomorphism $h:(M, R) \rightarrow\left(M^{\prime}, R^{\prime}\right)$ gets factored as

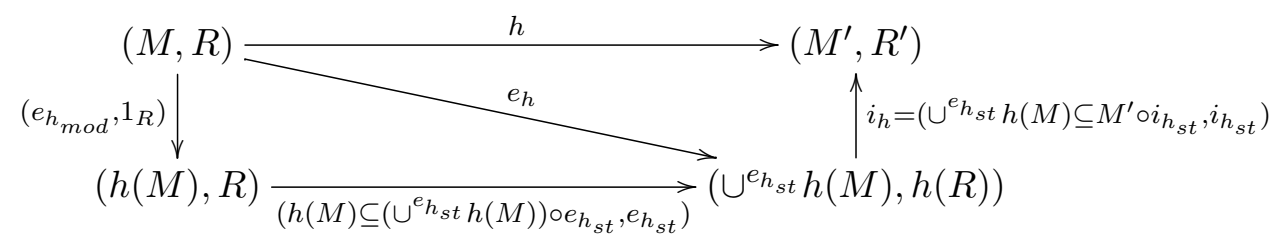

where

- $e_{h_{s t}}: R \rightarrow h(R)$ and $i_{h_{s t}}: h(R) \rightarrow R^{\prime}$ are the abstract surjection and the abstract inclusion that correspond to the factorization of $h_{s t}$,

$-e_{h_{m o d}}$ and $i_{h_{m o d}}$, respectively, consist of abstract surjections and abstract inclusions of $\Sigma$-models, respectively, and

- $\left(\cup^{e^{h_{s t}}} h(M), h(R)\right) \subseteq\left(M^{\prime} \circ i_{h_{s t}}, h(R)\right)$ is the unique extension of $i_{h_{m o d}}$ given by the universal property of $\left(\cup^{e_{s t}} h(M), h(R)\right)$.
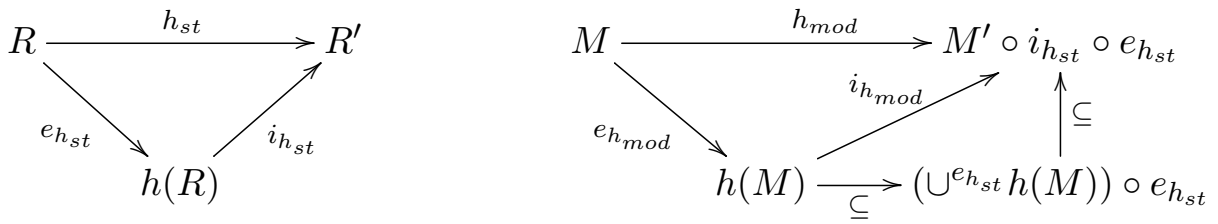

Obviously $\left(\cup^{e_{h t}} h(M) \subseteq M^{\prime} \circ i_{h_{s t}}, i_{h_{s t}}\right)$ is an abstract inclusion. It is also straightforward to check that $\left(e_{h_{m o d}} ;\left(h(M) \subseteq\left(\cup^{e_{h s t}} h(M)\right) \circ e_{h_{s t}}\right), e_{h_{s t}}\right)$ is an abstract surjection. By the conditions of the theorem we successively obtain that $\left(e_{h_{\text {mod }}}, 1_{R}\right),\left(h(M) \subseteq\left(\cup^{e_{s t}} h(M)\right) \circ e_{h_{s t}}, e_{h_{s t}}\right)$, and $\left(\cup^{e_{h t}} h(M) \subseteq M^{\prime} \circ i_{h_{s t}}, i_{h_{s t}}\right)$ are $\mathrm{Mod}^{\prime}$-homomorphisms, which implies that our factorization of $h$ happens inside $\operatorname{Mod}^{\prime}$.

In order to show the uniqueness property of the factorization let us assume a factorization

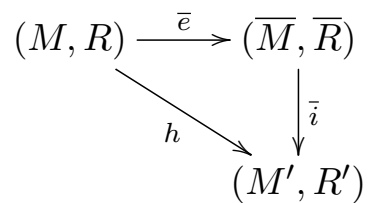

with $\bar{e}$ abstract surjection and $\bar{i}$ abstract inclusion and prove that $\bar{e}=e_{h}$ and $\bar{i}=i_{h}$. By the uniqueness of factorization in the category of (Nom, $\Lambda$ )-models we get immediately that $\bar{e}_{s t}=e_{h_{s t}}, \bar{i}_{s t}=i_{h_{s t}}$, and that $\bar{R}=h_{s t}(R)$. Since $\bar{i}_{\text {mod }}$ consists of inclusions, by the uniqueness of the factorizations in through $\left(I^{\Sigma}, \mathcal{E}^{\Sigma}\right)$ we get $\bar{e}(M)=h(M)$

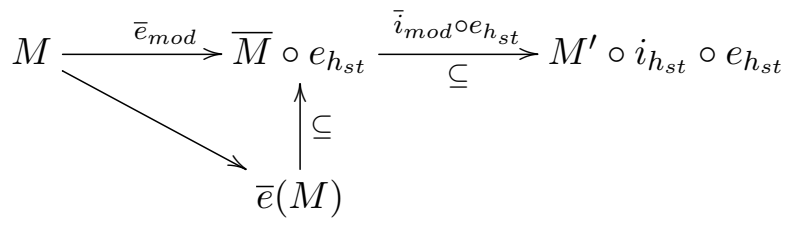

hence $\bar{M}=\cup^{e^{h_{s t}}} \bar{e}(M)=\cup^{e^{h_{s t}}} h(M)$. It follows that $\bar{e}_{\text {mod }}=\left(e_{h}\right)_{\text {mod }}$ and that $\bar{i}_{\text {mod }}=\left(i_{h}\right)_{\text {mod }}$.

The inclusion system for $\operatorname{Mod}^{\prime}(\Sigma$, Nom, $\Lambda)$ resulting from Thm. 5.1 may arise as a particular example of a 'Grothendieck inclusion system' of [16]; however this requires category theory concepts and skills much beyond the level of category theory of this paper which is meant to be rather elementary. 
Example 5.3 ( $\mathcal{H} P L$ sub-models). Given a $\mathcal{H} P L$-signature $(P, \mathrm{Nom}, \Lambda)$ we consider any of the strong or of the closed inclusion system for the (Nom, $\Lambda$ )-models and the strong inclusion system for the $P$-models. Checking the first two conditions of Thm. 5.1 is rather straightforward. For the third condition, given $h:(M, R) \rightarrow\left(M^{\prime}, R^{\prime}\right)$, for each $j \in\left|R^{\prime}\right|$ we define $\left(\cup^{e_{s t}} M\right)_{j}=\cup_{h_{s t}(i)=j} M_{i}$.

If we considered the T, $\mathrm{S} 4, \mathrm{~S} 5$ variants of the standard version of $\mathcal{H} P L$ (see Ex. 3.1) then in order to fulfill the conditions of Thm. 5.1 we have to consider only the closed inclusion system for the (Nom, $\Lambda$ )models as the strong one would not do it since $h(R)$ may not fulfill the respective conditions for T, S4 or S5.

Example 5.4 ( $\mathcal{H} R E L^{\prime}$ sub-models). Given a $\mathcal{H} R E L$-signature $(\Sigma$, Nom, $\Lambda)$ we consider any of the strong or of the closed inclusion system for the (Nom, $\Lambda$ )-models and the strong inclusion system for the $\Sigma$ models. Checking the first two conditions of Thm. 5.1 is rather straightforward. For the third condition, given $h:(M, R) \rightarrow\left(M^{\prime}, R^{\prime}\right)$, we define

- for each $j \in|h(R)|$ and each $i \in|R|,\left|\left(\cup^{e_{h t}} M\right)_{j}\right|=\left|M_{i}\right|$,

- for each constant $c$ in $\Sigma, j \in|h(R)|$ and $i \in|R|,\left(\left(\cup^{e_{s t}} M\right)_{j}\right)_{c}=\left(M_{i}\right)_{c}$, and

- for each relation symbol $\pi$ in $\Sigma$ and each $j \in|h(R)|,\left(\left(\cup^{e^{h_{s t}}} M\right)_{j}\right)_{\pi}=\cup_{h_{s t}(i)=j}\left(M_{i}\right)_{\pi}$.

For the case of the $\mathrm{T}, \mathrm{S} 4, \mathrm{~S} 5$ variants of the standard version of $\mathcal{H} R E L^{\prime}$ we have the same restriction as discussed in Ex. 5.3, namely that we can consider only the closed inclusion system for the (Nom, $\Lambda$ )models.

Example 5.5 ( $\mathcal{H} P A^{\prime}$ sub-models). Given a $\mathcal{H} P A$-signature $(\Sigma$, Nom, $\Lambda)$ we consider any of the strong or of the closed inclusion system for the (Nom, $\Lambda$ )-models and the strong inclusion system for the $\Sigma$-models. Let us check the conditions of Thm. 5.1 in some detail.

1. Since for all $i, j \in|R|$, on the one hand $M_{i}$ and $M_{j}$, and on the other hand $M_{h_{s t}(i)}^{\prime}$ and $M_{h_{s t}(j)}^{\prime}$, share the same total algebra part and also $h_{i}=h_{j}$ it follows that $h_{i}\left(M_{i}\right)$ and $h_{j}\left(M_{j}\right)$ share the same total algebra part too and that $e_{h_{i}}=e_{h_{j}}$.

From the factorization of $h_{i}$ through the strong inclusion system of $\Sigma$-models we have that for each partial operation symbol $\sigma$ and each adequate list of arguments $m, h_{i}\left(M_{i}\right)_{\sigma}(m)$ is defined if and only if there exists $m_{0} \in h_{i}^{-1}(m)$ such that $\left(M_{i}\right)_{\sigma}\left(m_{0}\right)$ is defined and moreover $h_{i}\left(M_{i}\right)_{\sigma}(m)=$ $h_{i}\left(\left(M_{i}\right)_{\sigma}\left(m_{0}\right)\right)$. It is straightforward to check that this definition is independent of the choice of $m_{0}$. In order to complete the checking of this condition we have establish that $(h(M), R)$ is a Mod'-model which amounts to showing that for all $i, j \in|R|$, we have that

$$
h_{i}\left(M_{i}\right)_{\sigma}(m)=h_{j}\left(M_{j}\right)_{\sigma}(m) \text { when both are defined. }
$$

We know that

$$
\begin{aligned}
& h_{k}\left(M_{k}\right)_{\sigma}(m)=h_{k}\left(\left(M_{k}\right)_{\sigma}\left(m_{k}\right)\right) \text { for some } m_{k} \in h_{k}^{-1}(m), k \in\{i, j\} \\
& h_{k}\left(\left(M_{k}\right)_{\sigma}\left(m_{k}\right)\right)=\left(M_{h_{s t}}^{\prime}\right)_{\sigma}\left(h_{k}\left(m_{k}\right)\right), k \in\{i, j\} .
\end{aligned}
$$

Since $h_{k}\left(m_{k}\right)=m$ and since $\left(M^{\prime}, R^{\prime}\right)$ is a Mod'-model we have that (6) follows from (7) and (8).

2. The second condition of Thm. 5.1 is straightforward.

3. For each $j \in|h(R)|,\left(\cup^{e_{s t}} M\right)_{j}$ is defined as follows:

- We let the total algebra part of $\left(\cup^{e_{h s}} M\right)_{j}$ to be that of $M_{i}$ for any $i \in|R|$. Note the this does not depend on the choice of $i$ because $(M, R)$ is Mod'-model. $^{\prime}$ - 
- For each partial operation symbol $\sigma$ of $\Sigma$ and each adequate list of arguments $m,\left(\left(\cup^{e_{h}}{ }_{s t} M\right)_{j}\right)_{\sigma}(m)$ is defined if and only if there exists $i \in h_{s t}^{-1}(j)$ such that $\left(M_{i}\right)_{\sigma}(m)$ is defined, and moreover $\left(\left(\cup^{e_{h s}} M\right)_{j}\right)_{\sigma}(m)=\left(M_{i}\right)_{\sigma}(m)$. Note that according to the definition of constrained $\mathcal{H} P A$ models in Ex. 3.4, $\left(\left(\cup^{e^{h}}{ }_{s t} M\right)_{j}\right)_{\sigma}(m)$ is well-defined.

Example 5.6 ( $\mathcal{H} A L G^{\prime}$ sub-models). Given a $\mathcal{H} A L G^{\prime}$-signature $(\Sigma$, Nom) we consider any of the strong or of the closed inclusion system for the (Nom, $\Lambda$ )-models and the standard inclusion system for the $\Sigma$ models. Let us check the conditions of Thm. 5.1 in some detail.

1. For this condition we essentially have to show that for each $(i, j) \in R_{\leq}$we have $h_{i}\left(M_{i}\right) \subseteq h_{j}\left(M_{j}\right)$. From $(i, j) \in R_{\leq}$it follows that $\left(h_{s t}(i), h_{s t}(j)\right) \in R_{\leq}^{\prime}$, hence $M_{h_{s t}(i)}^{\prime} \subseteq M_{h_{s t}(j)}^{\prime}$. By the DiagonalFill Lemma for inclusion systems (see [14]) there exists a homomorphism $h_{i}\left(M_{i}\right) \rightarrow h_{j}\left(M_{j}\right)$ such that the diagram below commutes; from the uniqueness of factorizations it follows that this is an inclusion.

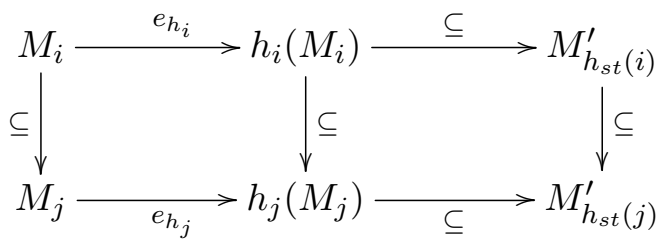

2. The second condition of Thm. 5.1 is straightforward.

3. Let us first note that since $R_{\leq}$and $R_{\leq}^{\prime}$ are complete lattices it follows that $h(R)_{\leq}$is a complete lattice too. For each $j \in|h(R)|,\left(\cup^{e} h_{s t} M\right)_{j}$ is defined as $\left.M_{\text {sup }\{i \mid} h_{s t}(i)=j\right\}$.

For each $\left(f, 1_{R}\right):(M, R) \rightarrow\left(N^{\prime} \circ e_{h_{s t}}, R\right)$ we let $f_{j}^{\prime}=f_{\text {sup }\left\{i \mid h_{s t}(i)=j\right\}}$.

\subsection{Preservation by sub-models}

Definition 5.3. In any institution $\mathcal{I}$ equipped with an inclusion system for each of its categories of models, a $\Sigma$-sentence $\rho$ is preserved by sub-models when for each sub-model $M^{\prime} \subseteq M, M \models \rho$ implies $M^{\prime} \models \rho$.

Example 5.7. The following preservation properties in $P L, R E L, A L G, P A$ are simple exercises and moreover they are known from the literature (e.g. [14], etc.):

- the negations of atoms are preserved by the plain sub-models,

- the atoms are preserved by the closed sub-models,

- the equational atoms are preserved by the plain sub-models in $R E L$ and $A L G$,

- the sentences preserved by the plain sub-models are closed under conjunction $\wedge$, disjunction $\vee$, and universal quantifications, and

- if $\rho_{1}$ is preserved along abstract inclusions and $\rho_{2}$ is preserved by sub-models then $\rho_{1} \Rightarrow \rho_{2}$ is preserved by sub-models.

Definition 5.4 (Local preservation by sub-models). In any hybridization of $\mathcal{I}, a(\Sigma$, Nom, $\Lambda)$-sentence $\rho$ is locally preserved by sub-models when for each sub-model $\left(M^{\prime}, R^{\prime}\right) \subseteq(M, R)$ and each $s \in\left|R^{\prime}\right|$, $(M, R) \models{ }^{s} \rho$ implies $\left(M^{\prime}, R^{\prime}\right) \models{ }^{s} \rho$.

Proposition 5.1. The sentences that are locally preserved by sub-models are also preserved by sub-models. 
Proof. Let $\left(M^{\prime}, R^{\prime}\right) \subseteq(M, R)$ be a sub-model relationship and let us assume that $(M, R) \models \rho$. For any $s \in\left|R^{\prime}\right|$, since $\left|R^{\prime}\right| \subseteq|R|$ we have $s \in|R|$. From $(M, R) \mid=\rho$ it follows that $(M, R) \mid={ }^{s} \rho$. Since $\rho$ is locally preserved by sub-models it follows that $\left(M^{\prime}, R^{\prime}\right) \models^{s} \rho$.

Definition 5.5. A signature morphism $\varphi: \Sigma \rightarrow \Sigma^{\prime}$ in an institution with inclusion systems for its categories of models lifts forward (backwards) inclusions when for any $\Sigma$-models $M \subseteq N$ and any $\varphi$-expansion $M^{\prime}$ $\left(N^{\prime}\right)$ of $M(N)$ there exists a $\varphi$-expansion $N^{\prime}\left(M^{\prime}\right)$ of $N(M)$ such that $M^{\prime} \subseteq N^{\prime}$.

Theorem 5.2. In any hybridization of an institution $\mathcal{I}$, that admits an inclusion system as described in Thm. 5.1:

1. Each nominal is locally preserved by sub-models.

2. If $\rho \in \operatorname{Sen}^{\mathcal{I}}(\Sigma)$ is preserved by sub-models in $\mathcal{I}$ then $\rho$ is locally preserved by sub-models in the hybridization.

3. If $\rho_{1}$ and $\rho_{2}$ are locally preserved by sub-models then $\rho_{1} \wedge \rho_{2}$ and $\rho_{1} \vee \rho_{2}$ are also locally preserved by sub-models.

4. If $\rho_{1}$ is locally preserved along inclusions and $\rho_{2}$ is locally preserved by sub-models then $\rho_{1} \Rightarrow \rho_{2}$ is locally preserved by sub-models.

5. If $\rho$ is locally preserved by sub-models then each $@_{i} \rho$ is also locally preserved by sub-models.

6. If $\rho$ are locally preserved by sub-models then $[\lambda](\rho)$ is also locally preserved by sub-models.

7. If $\chi$ lifts forward (backwards) inclusions and $\rho$ is locally preserved by sub-models then $(\forall \chi) \rho((\exists \chi) \rho)$ is locally preserved by sub-models.

Proof. Let us fix two $(\Sigma$, Nom, $\Lambda)$-models $(M, R)$ and $\left(M^{\prime}, R^{\prime}\right)$ such that $\left(M^{\prime}, R^{\prime}\right) \subseteq(M, R)$ with respect to the inclusion system resulting from Thm. 5.1 and $s \in\left|R^{\prime}\right|$.

1. Assume $(M, R) \models^{s} i$. This means $s=R_{i}$. Since $R^{\prime}$ is sub-model of $R$ (in $R E L$ ) we have that $R_{i}=R_{i}^{\prime}$. It follows that $s=R_{i}^{\prime}$ which implies $\left(M^{\prime}, R^{\prime}\right) \models^{s} i$.

2. Assume $(M, R) \models{ }^{s} \rho$. This means $M_{s} \mid=^{\mathcal{I}} \rho$. By the hypothesis that $\rho$ is preserved by sub-models in $\mathcal{I}$ it follows that $M_{s}^{\prime} \models^{\mathcal{I}} \rho$ which means $\left(M^{\prime}, R^{\prime}\right) \models{ }^{s} \rho$.

3. Assume $(M, R) \models{ }^{s} \rho_{1} \wedge \rho_{2}$. This means that $(M, R) \models{ }^{s} \rho_{1}$ and that $(M, R) \models{ }^{s} \rho_{2}$. Since both $\rho_{1}$ and $\rho_{2}$ are locally preserved by sub-models it follows that $\left(M^{\prime}, R^{\prime}\right) \models^{s} \rho_{1}$ and that $\left(M^{\prime}, R^{\prime}\right) \models{ }^{s} \rho_{2}$, hence $\left(M^{\prime}, R^{\prime}\right) \models^{s} \rho_{1} \wedge \rho_{2}$.

A similar argument may be developed for showing that $\rho_{1} \vee \rho_{2}$ is locally preserved by sub-models.

4. Assume $(M, R) \models{ }^{s} \rho_{1} \Rightarrow \rho_{2}$. We want to obtain that $\left(M^{\prime}, R^{\prime}\right) \models^{s} \rho_{1} \Rightarrow \rho_{2}$. Let us assume that $\left(M^{\prime}, R^{\prime}\right) \models{ }^{s} \rho_{1}$. Since $\rho_{1}$ is locally preserved along inclusions it follows that $(M, R) \models^{s} \rho_{1}$, hence $(M, R) \models{ }^{s} \rho_{2}$. Since $\rho_{2}$ is locally preserved by sub-models we obtain that $\left(M^{\prime}, R^{\prime}\right) \models^{s} \rho_{2}$.

5. Assume $(M, R) \models \models^{s} @_{i} \rho$. This means $(M, R) \models=^{R_{i}} \rho$. Since $R^{\prime}$ is sub-model of $R$ (in $R E L$ ) we have that $R_{i}=R_{i}^{\prime}$. Because $\rho$ is locally preserved by sub-models it follows that $\left(M^{\prime}, R^{\prime}\right) \models R_{i}^{\prime} \rho$, hence $\left(M^{\prime}, R^{\prime}\right) \models^{s} @_{i} \rho$.

6. Assume $(M, R) \models^{s}[\lambda]\left(\rho_{1}, \ldots, \rho_{n}\right)$. We want to obtain that for each $\left(s, s_{1}, \ldots, s_{n}\right) \in R_{\lambda}^{\prime}$, there exists $1 \leq k \leq n$ such that $\left(M^{\prime}, R^{\prime}\right) \models{ }^{s_{k}} \rho$. Since $R^{\prime}$ is sub-model of $R$ (in $R E L$ ) we have that $R_{\lambda}^{\prime} \subseteq R_{\lambda}$, hence $\left(s, s_{1}, \ldots, s_{n}\right) \in R_{\lambda}$. Thus there exists $1 \leq k \leq n$ such that $(M, R) \models{ }^{s_{k}} \rho_{k}$. Because $\rho_{k}$ is locally preserved by sub-models it follows that $\left(M^{\prime}, R^{\prime}\right) \models{ }^{s^{\prime}} \rho_{k}$.

7. Assume $(M, R) \models^{s}(\forall \chi) \rho$. We want to obtain that $\left(M^{\prime}, R^{\prime}\right) \models^{s}(\forall \chi) \rho$. Let $\left(\overline{M^{\prime}}, \overline{R^{\prime}}\right)$ be any $\chi$ expansion of $\left(M^{\prime}, R^{\prime}\right)$. By the forward lifting hypothesis there exists a $\chi$-expansion $(\bar{M}, \bar{R})$ of $(M, R)$ such 
that $\left(\overline{M^{\prime}}, \overline{R^{\prime}}\right) \subseteq(\bar{M}, \bar{R})$. Since $(\bar{M}, \bar{R}) \models^{s} \rho$ and because $\rho$ is locally preserved by sub-models it follows that $\left(\overline{M^{\prime}}, \overline{R^{\prime}}\right) \models^{s} \rho$.

Now we assume that $(M, R) \models^{s}(\exists \chi) \rho$ and under the backwards lifting hypothesis we prove that $\left(M^{\prime}, R^{\prime}\right) \models^{s}(\exists \chi) \rho$. There exists a $\chi$-expansion $(\bar{M}, \bar{R})$ of $(M, R)$ such that $(\bar{M}, \bar{R}) \models^{s} \rho$. By the backwards lifting hypothesis there exists a $\chi$-expansion $\left(\overline{M^{\prime}}, \overline{R^{\prime}}\right)$ of $\left(M^{\prime}, R^{\prime}\right)$ such that $\left(\overline{M^{\prime}}, \overline{R^{\prime}}\right) \subseteq(\bar{M}, \bar{R})$. Because $\rho$ is locally preserved by sub-models it follows that $\left(\overline{M^{\prime}}, \overline{R^{\prime}}\right) \models{ }^{s} \rho$, hence $\left(M^{\prime}, R^{\prime}\right) \models^{s}(\exists \chi) \rho$.

Example 5.8. The last two items of Ex. 5.7 may be derived from the previous items of Ex. 5.7 via the result of Thm. 5.2 by considering $|R|$ and $\left|R^{\prime}\right|$ singleton sets.

The following examples of preservation by sub-models may be established by recursion on the structure of the sentences by application of Thm. 5.2 and at the end by application of Prop. 5.1. In the application of Thm. 5.2, the $\Rightarrow$ steps require the application of Prop. 4.1. The base steps require preservation results in corresponding base institutions; these may be found in Ex. 5.7 and in Ex. 4.1. The steps corresponding to the quantifications are justified by noting that in all our examples the signature extensions with nominal variables and with base institution variables lift forward inclusions.

- In HPL: $(\forall i, j)\left(\left(\left(@_{i} \pi\right) \wedge\left(@_{j} \pi\right)\right) \Rightarrow @_{i} \square j\right)$.

- In $\mathcal{H} R E L^{\prime}:(\forall x, y)(\pi(x, y) \Rightarrow[\lambda](x=y))$.

- In $\mathcal{H} A L G^{\prime}: @_{i}(\varnothing X) t=t^{\prime}$.

- In $\mathcal{H} A L G^{\prime}:(\forall X)\left(\left(t_{1}=t_{2}\right) \Rightarrow \square(\forall Y) t_{1}^{\prime}=t_{2}^{\prime}\right)$.

\section{Products of models in hybridized institutions}

This section consists of two parts:

1. We develop a method to establish the existence of products of models in hybridized institutions.

2. We develop results about preservation by products in hybridized institutions.

\subsection{Existence of products of models in hybridized institutions}

The following result may be obtained as a particular case of the general result on existence of limits in Grothendieck categories of [36]. We give it here an explicit construction and proof that is needed in the applications and in the examples.

Proposition 6.1. If $\operatorname{Mod}^{\mathcal{I}}(\Sigma)$ has products then $\operatorname{Mod}^{\mathcal{H I}}(\Sigma, \mathrm{Nom}, \Lambda)$ has products of models as follows. For each family $\left\{\left(M^{i}, R^{i}\right) \mid i \in I\right\}$ of $(\Sigma$, Nom, $\Lambda)$-models its product $(M, R)$ is defined by

- $R=\prod_{i \in I} R^{i}$ (product of (Nom, $\Lambda$ )-models), and

- for each $k=\left(k^{i}\right)_{i \in I} \in|R|=\prod_{i \in I}\left|R^{i}\right|, M_{k}=\prod_{i \in I} M_{k^{i}}^{i}$ (product of $\Sigma$-models).

Proof. We define the projections $\left\{p^{i}:(M, R) \rightarrow\left(M^{i}, R^{i}\right) \mid i \in I\right\}$ as follows.

- $p_{s t}^{i}$ is the projection $R \rightarrow R^{i}$ in the category of the (Nom, $\Lambda$ )-models; note that $|R|=\prod_{i \in I}\left|R^{i}\right|$, that for each $n \in$ Nom, $R_{n}=\left(R_{n}^{i}\right)_{i \in I}$, and that for each $\lambda \in \Lambda_{n}$ we have that $R_{\lambda}=\left\{\left(s_{1}, \ldots, s_{n}\right) \mid\right.$ $\left.\left(s_{1}^{i}, \ldots, s_{n}^{i}\right) \in R_{\lambda}^{i}, i \in I\right\}$, and

- $p_{\text {mod }}^{i}: M \rightarrow M^{i} \circ p_{s t}^{i}$ is defined such that for each $k \in|R|,\left(p_{\text {mod }}^{i}\right)_{k}: M_{k} \rightarrow M_{k^{i}}^{i}$ is the corresponding projection in the category of the $\Sigma$-models. 
For any family of $(\Sigma$, Nom, $\Lambda)$-model homomorphisms $\left\{h^{i}:\left(M^{\prime}, R^{\prime}\right) \rightarrow\left(M^{i}, R^{i}\right) \mid i \in I\right\}$ there exists an unique $h:\left(M^{\prime}, R^{\prime}\right) \rightarrow(M, R)$ defined as follows:

- $h_{s t}$ is the unique homomorphism of (Nom, $\Lambda$ )-models such that for each $i \in I, h_{s t} ; p_{s t}^{i}=h_{s t}^{i}$, and

- for each $j \in\left|R^{\prime}\right|, h_{j}$ is the unique homomorphism of $\Sigma$-models such that for each $i \in I, h_{j} ;\left(p^{i}\right)_{h_{s t}(j)}=$ $\left(h^{i}\right)_{j}$.
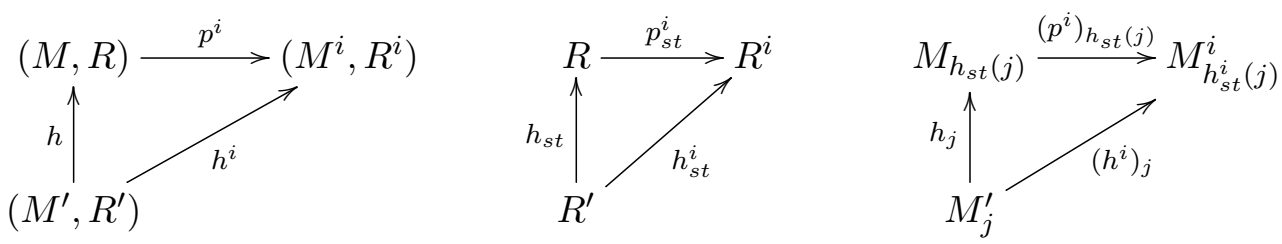

Prop. 6.1 develops products of non-constrained models and may be applied directly only to Ex. 6.1 below. However often products of constrained models may be obtained as designated products of nonconstrained models as given by Prop. 6.1; this is the case for Ex. $6.2\left(\mathcal{H} R E L^{\prime}\right)$, Ex. $6.3\left(\mathcal{H} P A^{\prime}\right)$, and Ex. 6.4 $\left(\mathcal{H} A L G^{\prime}\right)$ below.

Example 6.1 (Products of $\mathcal{H} P L$-models). Recall that in $P L$, the product of a family $\left\{M^{i} \mid i \in I\right\}$ of $P$-models is given by the intersection $\cap_{i \in I} M^{i}$.

Hence by Prop. 6.1, $\mathcal{H} P L$ has products of models as given by the construction described in Prop. 6.1.

Example 6.2 (Products of $\mathcal{H} R E L^{\prime}$-models). In Prop. 6.1 we have established that $R E L$ has products of models, hence again by Prop. 6.1 we obtain that $\mathcal{H} R E L$ has products of models. Let us call the products of models in $R E L$ that have been described in Prop. 6.1, cartezian products.

In order to establish that $\mathcal{H} R E L^{\prime}$ has products of models we show that for any family $\left\{\left(M^{i}, R^{i}\right) \mid\right.$ $i \in I\} \subseteq\left|\operatorname{Mod}^{\prime}(\Sigma, \operatorname{Nom}, \Lambda)\right|$ their product $(M, R)$ in $\operatorname{Mod}^{\mathcal{H} R E L}(\Sigma, \operatorname{Nom}, \Lambda)$ that is constructed from cartezian products of $\Sigma$-models, i.e. for each $k \in|R|, M_{k}$ is the cartezian product $\prod_{i \in I} M_{k^{i}}^{i}$, is a product in $\operatorname{Mod}^{\prime}(\Sigma, \operatorname{Nom}, \Lambda)$ :

- For any $j, k \in|R|$ we have the cartezian products of sets $\left|M_{j}\right|=\prod_{i \in I}\left|M_{j^{i}}^{i}\right|$ and $\left|M_{k}\right|=\prod_{i \in I}\left|M_{k^{i}}^{i}\right|$. Because each $\left(M^{i}, R^{i}\right)$ is a $\operatorname{Mod}^{\prime}$-model we have that $\left|M_{j^{i}}^{i}\right|=\left|M_{k^{i}}^{i}\right|$, hence because cartezian products are unique we get $\left|M_{j}\right|=\left|M_{k}\right|$.

- For any constant $c$ of $\Sigma$ and for any $j, k \in|R|$, because $\left(M_{j^{i}}^{i}\right)_{c}=\left(M_{k^{i}}^{i}\right)_{c}$ it follows that $\left(M_{j}\right)_{c}=$ $\left(\left(M_{j^{i}}^{i}\right)_{c}\right)_{i \in I}=\left(\left(M_{k^{i}}^{i}\right)_{c}\right)_{i \in I}=\left(M_{k}\right)_{c}$.

Hence $(M, R) \in\left|\operatorname{Mod}^{\prime}(\Sigma, \operatorname{Nom}, \Lambda)\right|$. It is straightforward to check further that the projections $p^{i}:(M, R) \rightarrow$ $\left(M^{i}, R^{i}\right)$ also belong to $\operatorname{Mod}^{\prime}(\Sigma, \operatorname{Nom}, \Lambda)$. Moreover, if each $h^{i}$ from the proof of Prop. 6.1 is in $\operatorname{Mod}^{\prime}(\Sigma, \operatorname{Nom}, \Lambda)$ then the mediating homomorphism $h$ is also in $\operatorname{Mod}^{\prime}(\Sigma, \operatorname{Nom}, \Lambda)$.

Example 6.3 (Products of $\mathcal{H} P A^{\prime}$-models). In $P A$, any family $\left\{M^{i} \mid i \in I\right\}$ of $\Sigma$-models has products as follows.

1. We consider the cartezian product of the underlying universes $|M|=\prod_{i \in I}\left|M^{i}\right|$ with projections $p^{i}:|M| \rightarrow\left|M^{i}\right|$. 
2. For each operation $\sigma$ of $\Sigma$ and $m$ adequate list of arguments, $M_{\sigma}(m)$ is defined if and only if for each $i \in I, M_{\sigma}^{i}\left(m^{i}\right)$ is defined ( $m^{i}$ denotes the list of arguments obtained from $m$ by applying the projection $p^{i}$ to each of the arguments). When $M_{\sigma}(m)$ is defined its value is $M_{\sigma}(m)=\left(M_{\sigma}^{i}\left(m^{i}\right)\right)_{i \in I}$.

Let us call the product defined above a cartezian product.

In order to establish that $\mathcal{H} P A^{\prime}$ has products of models we do the same as in Ex. 6.2 above, namely we show that for any family $\left\{\left(M^{i}, R^{i}\right) \mid i \in I\right\} \subseteq\left|\operatorname{Mod}^{\prime}(\Sigma, \operatorname{Nom}, \Lambda)\right|$ their product $(M, R)$ in $\operatorname{Mod}^{\mathcal{H} P A}(\Sigma, \operatorname{Nom}, \Lambda)$ that is constructed from cartezian products of $\Sigma$-models, is a product in $\operatorname{Mod}^{\prime}(\Sigma, \operatorname{Nom}, \Lambda)$. The constraint on the underlying universes of $(M, R)$ may be checked as in Ex. 6.2 above. For the other constraint on $(M, R)$ let us consider $j, k \in|R|, \sigma$ operation in $\Sigma$ and $m$ adequate list of arguments and let us assume that both $\left(M_{j}\right)_{\sigma}(m)$ and $\left(M_{k}\right)_{\sigma}(m)$ are defined. This implies that for each $i \in I$, both $\left(M_{j^{i}}^{i}\right)_{\sigma}\left(m^{i}\right)$ and $\left(M_{k^{i}}^{i}\right)_{\sigma}\left(m^{i}\right)$ are defined. Since $\left(M^{i}, R^{i}\right)$ is a Mod'-model it follows that $\left(M_{j^{i}}^{i}\right)_{\sigma}\left(m^{i}\right)=\left(M_{k^{i}}^{i}\right)_{\sigma}\left(m^{i}\right)$. Hence $\left(M_{j}\right)_{\sigma}(m)=\left(M_{k}\right)_{\sigma}(m)$.

Example 6.4 (Products of $\mathcal{H} A L G^{\prime}$-models). In order to establish that $\mathcal{H} A L G^{\prime}$ has products of models we first note that the products of complete lattices yield complete lattices and then we proceed as in Ex. 6.2 and Ex. 6.3 above. More precisely, we show that the product $(M, R)$ of any family $\left\{\left(M^{i}, R^{i}\right) \mid i \in I\right\} \subseteq$ $\left|\operatorname{Mod}^{\prime}(\Sigma, \operatorname{Nom})\right|$ in $\operatorname{Mod}^{\mathcal{H} A L G}(\Sigma, \operatorname{Nom}, \Lambda)$ that is constructed from cartezian products of $\Sigma$-models, is a product in $\operatorname{Mod}^{\prime}(\Sigma$, Nom $)$. The specific constraint on $(M, R)$, namely that if $(j, k) \in R_{\leq}$then $M_{j}$ is a subalgebra of $M_{k}$, follows easily from the fact that for each $i \in I$, we have that $\left(j^{i}, k^{i}\right) \in R_{\leq}^{i}$ which implies that $M_{j^{i}}^{i}$ is a sub-algebra of $M_{k^{i}}^{i}$, hence the cartezian product $\prod_{i \in I} M_{j^{i}}^{i}$ is a sub-algebra of the cartezian product $\prod_{i \in I} M_{k^{i}}^{i}$. The corresponding property for projections and for the mediating homomorphisms follow easily; we skip these here.

\subsection{Preservation by products}

Definition 6.1. In any institution a sentence $\rho$ is preserved by products when for each product of models $\prod_{i \in I} M_{i}$ if for each $i \in I, M_{i}=\rho$ then $\prod_{i \in I} M_{i} \models \rho$.

Example 6.5. In the institutions $P L, R E L, A L G$ and $P A$ the following preservation are simple exercises; moreover they are well known from the literature (e.g. [14], etc.):

- all atoms are preserved by products,

- the sentences preserved by products are closed under conjunction $\wedge$ and universal and existential quantifications, and

- if $\rho_{1}$ is preserved along product projections and $\rho_{2}$ is preserved by products then $\rho_{1} \Rightarrow \rho_{2}$ is preserved by products.

Definition 6.2 (Local preservation by products). In any hybridization of an institution $\mathcal{I}, a(\Sigma$, Nom, $\Lambda)$ sentence $\rho$ is locally preserved by products when for each product $(M, R)$ of a family of $(\Sigma, N o m, \Lambda)$ models $\left\{\left(M^{i}, R^{i}\right) \mid i \in I\right\}$ and each $s \in|R|,\left\{\left(M^{i}, R^{i}\right) \models p_{s t}^{i}(s) \rho \mid i \in I\right\}$ implies $(M, R) \models{ }^{s} \rho$.

Without loss of generality in what follows we assume that $R$ is the cartezian product of $\left\{\left(M^{i}, R^{i}\right) \mid i \in I\right\}$.

Proposition 6.2. The sentences that are locally preserved by products are also preserved by products. 
Proof. Let us consider a product $(M, R)$ of a family $\left\{\left(M^{i}, R^{i}\right) \mid i \in I\right\}$. Without loss of generality we may assume that $R$ is the cartezian product of $\left\{R^{i} \mid i \in I\right\}$. Let us assume that for each $i \in I,\left(M^{i}, R^{i}\right) \models \rho$. Then for each $s=\left(s^{i}\right)_{i \in I} \in|R|$, we have that for each $i \in I,\left(M^{i}, R^{i}\right) \mid=^{s^{i}} \rho$. Because $\rho$ is locally preserved by products it follows that $(M, R) \models^{s} \rho$.

Definition 6.3. In an institution a signature morphism $\chi: \Sigma \rightarrow \Sigma^{\prime}$ lifts products forward (backwards) when for each product of $\Sigma$-models $\left\{p_{i}: \prod_{i \in I} M_{i} \rightarrow M_{i} \mid i \in I\right\}$ and each expansion(s) $M^{\prime}\left(M_{i}^{\prime}, i \in I\right)$ of $\prod_{i \in I} M_{i}\left(M_{i}, i \in I\right)$ there exists a x-expansion $\left\{p_{i}^{\prime}: \prod_{i \in I} M_{i}^{\prime} \rightarrow M_{i}^{\prime} \mid i \in I\right\}$ of $\left\{p_{i}: \prod_{i \in I} M_{i} \rightarrow\right.$ $\left.M_{i} \mid i \in I\right\}$ which is a product.

\section{Theorem 6.1.}

1. Each nominal is locally preserved by products.

2. If $\rho \in \operatorname{Sen}^{\mathcal{I}}(\Sigma)$ is preserved by products in the base institution $\mathcal{I}$ then $\rho$ is locally preserved by products in the hybridization.

3. If $\rho_{1}$ and $\rho_{2}$ are locally preserved by products then $\rho_{1} \wedge \rho_{2}$ is also locally preserved by products.

4. If $\rho_{1}$ is locally preserved along product projections and $\rho_{2}$ is locally preserved by products then $\rho_{1} \Rightarrow \rho_{2}$ is locally preserved by products.

5. If $\rho$ is locally preserved by products then each $@_{j} \rho$ is also locally preserved by products.

6. If $\rho$ is locally preserved by products and $\lambda$ is a binary modality, then $[\lambda](\rho)$ is locally preserved by products.

7. If $\rho$ are locally preserved by products, then $\langle\lambda\rangle(\rho)$ is also locally preserved by products.

8. If $\chi$ lifts forward (backwards) products and $\rho$ is locally preserved by products then $(\forall \chi) \rho((\exists \chi) \rho)$ is locally preserved by products.

Proof. Let $(M, R)$ be a product $\prod_{i \in I}\left(M^{i}, R^{i}\right)$ of $(\Sigma$, Nom, $\Lambda)$-models with projections $p^{i}:(M, R) \rightarrow$ $\left(M^{i}, R^{i}\right), i \in I$, and let $s \in|R|$ with $s=\left(s^{i}\right)_{i \in I}$ (where $s^{i}=p_{s t}^{i}(s)$ ).

1. Assume $\left(M^{i}, R^{i}\right) \models \models^{s^{i}} n \in$ Nom, $i \in I$. This means $\left(R^{i}\right)_{n}=s^{i}, i \in I$. It follows that $R_{n}=$ $\left(\left(R^{i}\right)_{n}\right)_{i \in I}=\left(s^{i}\right)_{i \in I}=s$. Hence $(M, R) \models{ }^{s} n$.

2. Assume $\left(M^{i}, R^{i}\right) \models s^{s^{i}} \rho, i \in I$, for $\rho \in \operatorname{Sen}^{\mathcal{I}}(\Sigma)$. This means $M_{s^{i}}^{i} \models^{\mathcal{I}} \rho, i \in I$. Since $\rho$ is preserved by products in $\mathcal{I}$ it follows that $M_{s} \models^{\mathcal{I}} \rho$ hence $(M, R) \models^{s} \rho$.

3. Assume $\left(M^{i}, R^{i}\right) \models=^{s^{i}} \rho_{1} \wedge \rho_{2}, i \in I$. This means $\left(M^{i}, R^{i}\right) \models \models^{s^{i}} \rho_{k}, i \in I, k \in\{1,2\}$. Since $\rho_{k}$, $k \in\{1,2\}$, is locally preserved by products it follows that $(M, R) \models^{s} \rho_{k}, k \in\{1,2\}$, hence $(M, R) \models s$ $\rho_{1} \wedge \rho_{2}$.

4. Assume $\left(M^{i}, R^{i}\right) \models{ }^{s^{i}} \rho_{1} \Rightarrow \rho_{2}, i \in I$. We want to show that $(M, R) \mid=^{s} \rho_{1} \Rightarrow \rho_{2}$. If $(M, R) \mid=^{s} \rho_{1}$, since $\rho_{1}$ is locally preserved along product projections it follows that for each $i \in I,\left(M^{i}, R^{i}\right) \models=^{s^{i}} \rho_{1}$. Hence for each $i \in I,\left(M^{i}, R^{i}\right)==^{s^{i}} \rho_{2}$. Since $\rho_{2}$ is locally preserved by products it follows that $(M, R) \models s$ $\rho_{2}$.

5. Assume $\left(M^{i}, R^{i}\right) \models s^{i} @_{j} \rho, i \in I$. This means $\left(M^{i}, R^{i}\right) \models{ }^{i} \rho$. Since $R_{j}=\left(R_{j}^{i}\right)_{i \in I}$ by the local preservation hypothesis it follows that $(M, R) \models{ }^{R_{j}} \rho$. Hence $(M, R) \models{ }^{s} @_{j} \rho$.

6. Assume $\left(M^{i}, R^{i}\right) \models=^{i}[\lambda](\rho), i \in I$. We want to show that for each $s^{\prime}$ such that $\left(s, s^{\prime}\right) \in R_{\lambda}$, $(M, R) \models s^{\prime} \rho$. Then for each $i \in I,\left(s^{i}, s^{\prime i}\right) \in R_{\lambda}^{i}$ hence $\left(M^{i}, R^{i}\right) \models{ }^{s^{\prime i}} \rho$. Since $\rho$ is locally preserved by products, $(M, R) \models \models^{s^{\prime}} \rho$.

7. Assume $\left(M^{i}, R^{i}\right) \models s^{i}\langle\lambda\rangle(\rho)$. We want to show that there exists $s_{1}, \ldots, s_{n}$ such that $\left(s, s_{1}, \ldots, s_{n}\right) \in$ $R_{\lambda}$ and for each $1 \leq k \leq n,(M, R) \models{ }^{s_{k}} \rho$. By our assumption for each $i \in I$, there exists $s_{1}^{i}, \ldots, s_{n}^{i}$ such 
that $\left(s^{i}, s_{1}^{i}, \ldots, s_{n}^{i}\right) \in R_{\lambda}^{i}$ and such that for each $1 \leq k \leq n,\left(M^{i}, R^{i}\right) \models s_{k}^{i} \rho_{k}$. For each $1 \leq k \leq n$ we define $s_{k}=\left(s_{k}^{i}\right)_{i \in I}$. Then because each $\rho_{k}$ is locally preserved by products, it follows that $(M, R) \models{ }^{s_{k}} \rho_{k}$. Note that $\left(s, s_{1}, \ldots, s_{n}\right) \in R_{\lambda}$. Hence $(M, R) \models^{s}\langle\lambda\rangle\left(\rho_{1}, \ldots, \rho_{n}\right)$.

8. Assume $\left(M^{i}, R^{i}\right) \models{ }^{s^{i}}(\forall X) \rho, i \in I$. We want to show that $(M, R) \models^{s}(\forall X) \rho$. Let $(\bar{M}, \bar{R})$ be any $\chi$-expansion of $(M, R)$. Since $\chi$ lifts products forward, for each $i \in I$ there exists a $\chi$-expansion $\left(\overline{M^{i}}, \overline{R^{i}}\right)$ of $\left(M^{i}, R^{i}\right)$ such that $(\bar{M}, \bar{R})$ is a product of the family $\left\{\left(\overline{M^{i}}, \overline{R^{i}}\right) \mid i \in I\right\}$. Then for each $i \in I$, $\left(\overline{M^{i}}, \overline{R^{i}}\right) \models{ }^{s^{i}} \rho$ and because $\rho$ is locally preserved by products it follows that $(\bar{M}, \bar{R}) \models=^{s} \rho$.

Now let us assume $\left(M^{i}, R^{i}\right) \models^{s^{i}}(\exists X) \rho, i \in I$. We want to show that $(M, R) \models^{s}(\exists X) \rho$. For each $i \in I$ there exists $\left(\overline{M^{i}}, \overline{R^{i}}\right)$ a $\chi$-expansion of $\left(M^{i}, R^{i}\right)$ such that $\left(\overline{M^{i}}, \overline{R^{i}}\right) \models \models^{s^{i}} \rho$. Since $\chi$ lifts products backwards it follows that there exists a $\chi$-expansion $(\bar{M}, \bar{R})$ of $(M, R)$ which is a product of the family $\left\{\left(\overline{M^{i}}, \overline{R^{i}}\right) \mid i \in I\right\}$. Since $\rho$ is locally preserved by products it follows that $(\bar{M}, \bar{R}) \mid={ }^{s} \rho$, hence $\left.(M, R)\right|^{s}(\exists X) \rho$.

Example 6.6. The last two items of Ex. 6.5 may be derived from the previous items of Ex. 6.5 via the result of Thm. 6.1 by considering $|R|$ and $\left|R^{\prime}\right|$ singleton sets.

The following examples of preservation by sub-models may be established by recursion on the structure of the sentences by application of Thm. 6.1 and at the end by application of Prop. 6.2. In the application of Thm. 5.2, the $\Rightarrow$ steps require the application of Prop. 4.1. The base steps require preservation results in corresponding base institutions; these may be found in Ex. 6.5 and in Ex. 4.1. The steps corresponding to the quantifications are justified by noting that in all our examples the signature extensions with nominal variables and with base institution variables lift products forward.

$$
\begin{aligned}
& \text { - In HPL: }(\forall i, j)\left(\left(\left(@_{i} \pi\right) \wedge\left(@_{j} \pi\right)\right) \Rightarrow @_{i} \square j\right) \text {. } \\
& \text { - In HREL': }(\forall x, y)(\pi(x, y) \Rightarrow[\lambda](x=y)) \text {. } \\
& \text { - In } \mathcal{H} A L G^{\prime}: @_{i}(\forall X) t=t^{\prime} \text {. } \\
& \text { - In } \mathcal{H} A L G^{\prime}:(\forall X)\left(\left(t_{1}=t_{2}\right) \Rightarrow \square(\forall Y) t_{1}^{\prime}=t_{2}^{\prime}\right) \text {. }
\end{aligned}
$$

\section{Initial semantics in hybridized institutions}

In this section we join our results developed above for establishing initial semantics in hybridized institutions.

The following is a straightforward consequence of Prop. 5.1 and of Prop. 6.2.

Corollary 7.1. If $\rho$ is (locally) preserved by sub-models and products then $\rho^{*}$ is a quasi-variety.

At the heart of our method for establishing initial semantics for hybridized institutions lies the following abstract relationship between quasi-varieties and initiality. It may be found in various slightly different forms in the categorical literature on quasi-varieties; the form presented here is taken from [14].

Proposition 7.1. Consider a category $\mathbb{C}$ with an initial object $0_{\mathbb{C}}$, small products, and with a co-wellpowered epic inclusion system. Then each quasi-variety $\mathcal{Q}$ of $\mathbb{C}$ has an initial object $0_{\mathcal{Q}}$. Moreover the unique arrow $0_{\mathbb{C}} \rightarrow 0_{\mathcal{Q}}$ is abstract surjection.

The following two results deal with the special conditions on the inclusion systems required by Prop. 7.1. 
Proposition 7.2. The inclusion system for $\operatorname{Mod}^{\prime}(\Sigma, N o m, \Lambda)$ defined in Thm. 5.1 is epic when $\left(I^{\Sigma}, \mathcal{E}^{\Sigma}\right)$ is epic.

Proof. Let $h:(M, R) \rightarrow\left(M^{\prime}, R^{\prime}\right)$ and $f, g:\left(M^{\prime}, R^{\prime}\right) \rightarrow\left(M^{\prime \prime}, R^{\prime \prime}\right)$ in $\operatorname{Mod}^{\prime}(\Sigma, \operatorname{Nom}, \Lambda)$ such that $h ; f=h ; g$. We have to prove that $f=g$.

That $f_{s t}=g_{s t}$ follows by the epi property of $h_{s t}$ which is a surjective homomorphism of (Nom, $\Lambda$ )models.

For each $s \in|R|$, from $h_{s} ; f_{h_{s t}(s)}=h_{s} ; g_{h_{s t}(s)}$ and by the assumption that $\left(I^{\Sigma}, \mathcal{E}^{\Sigma}\right)$ is epic we have that $i_{h_{s}} ; f_{h_{s t}(s)}=i_{h_{s}} ; g_{h_{s t}(s)}$. Since $M^{\prime}=\cup^{h_{s t}} h(M)$, by the uniqueness part of the universal property of $\left(i_{h_{\text {mod }}}, 1_{R}\right)$ we get that $f_{\text {mod }}=g_{\text {mod }}$.
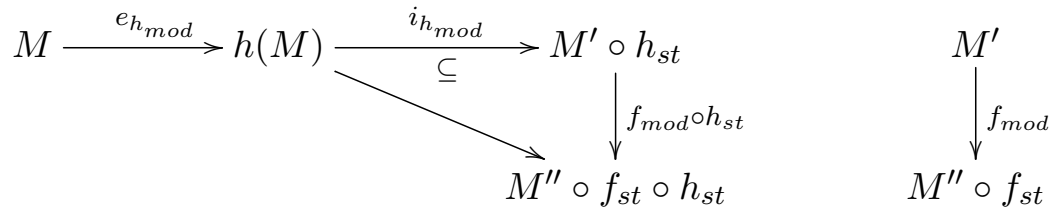

This completes the proof that $f=g$.

Example 7.1. From Ex. 5.2 and from Prop. 7.2 we obtain that the inclusion systems for $\mathcal{H} P L, \mathcal{H} R E L^{\prime}$, $\mathcal{H} P A^{\prime}, \mathcal{H} A L G^{\prime}$ are epic.

Proposition 7.3. The inclusion system for $\operatorname{Mod}^{\prime}(\Sigma, \operatorname{Nom}, \Lambda)$ defined in Thm. 5.1 is co-well-powered when $\left(I^{\Sigma}, \mathcal{E}^{\Sigma}\right)$ is co-well-powered.

Proof. For any model $(M, R)$ of the hybridization we may easily establish that

$$
\operatorname{card}(q(M, R)) \leq \operatorname{card}(q(R)) \times \operatorname{card}\left(\prod_{i \in|R|} q\left(M_{i}\right)\right)
$$

by noting that for each abstract surjection $h:(M, R) \rightarrow\left(M^{\prime}, R^{\prime}\right)$ when we factor each $h_{s}, s \in|R|$, through the inclusion system $\left(I^{\Sigma}, \mathcal{E}^{\Sigma}\right)$,

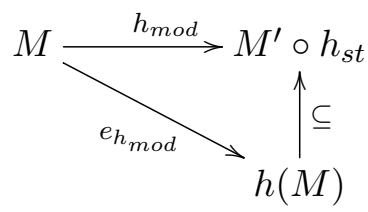

then $M^{\prime}=\cup^{h_{s t}} M$ is uniquely determined by $h(M)$.

Example 7.2. From Ex. 5.2 and from Prop. 7.3 we obtain that the inclusion systems for $\mathcal{H} P L, \mathcal{H} R E L^{\prime}$, $\mathcal{H} P A^{\prime}, \mathcal{H} A L G^{\prime}$ are co-well-powered.

The following initial semantics result is obtained directly by joining the results of Prop. 7.1, Cor. 7.1, Thm. 5.1, Fact 5.1, Prop. 7.2 and Prop. 7.3.

Corollary 7.2 (Initial semantics). Let us consider a signature $(\Sigma$, Nom, $\Lambda)$ in a hybridized institution $\left(\operatorname{Sign}^{\mathcal{H I}}, \operatorname{Sen}^{\mathcal{H I}}, \operatorname{Mod}^{\prime}, \mid=\right)$ such that $\operatorname{Mod}^{\prime}(\Sigma, \operatorname{Nom}, \Lambda)$ satisfies the following conditions: 
1. it has small products,

2. it has an epic and co-well powered inclusion system $\left(I^{\Sigma}, \mathcal{E}^{\Sigma}\right)$ for the category of $\Sigma$-models which together with a fixed choice between the strong and the closed inclusion systems for the (Nom, $\Lambda$ )models satisfies the conditions of Thm. 5.1, and

3. it has an initial model $0_{(\Sigma, \mathrm{Nom}, \Lambda)}$.

If each sentence in $E \subseteq \operatorname{Sen}^{\mathcal{H I}}(\Sigma$, Nom, $\Lambda)$ is (locally) preserved by sub-models and products then $E^{*}$ has an initial model $0_{E}$. Moreover, the unique model homomorphism $0_{(\Sigma, \mathrm{Nom}, \Lambda)} \rightarrow 0_{E}$ is an abstract surjection with respect to the inclusion system determined through Thm. 5.1.

Example 7.3. In order to apply Cor. 7.2 to our benchmark examples $\mathcal{H} P L, \mathcal{H} R E L^{\prime}, \mathcal{H} P A^{\prime}$ and $\mathcal{H} A L G^{\prime}$ it remains only to check the existence of $0_{(\Sigma, N o m, \Lambda)}$ as the other conditions have been discussed in examples above. In all these cases let us denote the presumed initial model $0_{(\Sigma, \mathrm{Nom}, \Lambda)}$ by $(M, R)$. Then:

- in $\operatorname{Mod}^{\mathcal{H} P L}(P, \mathrm{Nom}):|R|=\operatorname{Nom}, R_{\lambda}=\emptyset$, and for each $i \in$ Nom, $M_{i}=\emptyset$,

- in $\operatorname{Mod}^{\mathcal{H} R E L^{\prime}}(\Sigma$, Nom, $\Lambda):|R|=$ Nom, for each $\lambda \in \Lambda, R_{\lambda}=\emptyset$, and for each $i \in$ Nom, $\left|M_{i}\right|$ is the set of the constants of $\Sigma$ and for each relation symbol $\pi$ in $\Sigma,\left(M_{i}\right)_{\pi}=\emptyset$,

- in $\operatorname{Mod}^{\mathcal{H} P A^{\prime}}(\Sigma$, Nom, $\Lambda):|R|=$ Nom, for each modality symbol $\lambda$ in $\Lambda, R_{\lambda}=\emptyset$, and for each $i \in$ Nom, the underlying total algebra of $M_{i}$ is the initial term algebra for the total operation symbols and for each partial operation symbol $\sigma$ in $\Sigma, \operatorname{dom}\left(\left(M_{i}\right)_{\sigma}\right)=\emptyset$, and

- in $\operatorname{Mod}^{\mathcal{H} A L G^{\prime}}\left(\Sigma\right.$, Nom): $|R|=\operatorname{Nom} \cup\{\perp, \top\}, R_{\leq}=\{(\perp, n),(n, \top) \mid n \in \operatorname{Nom}\}, R_{\lambda}=\emptyset$, and for each $i \in|R|$, the underlying total algebra of $M_{i}$ is the initial $\Sigma$-algebra.

Example 7.4. In the concrete situations of interest one obstacle for the application of the initial semantics result given by Cor. 7.2 is related to the application of Thm. 5.2. The notion of sub-model determined by Thm. 5.1 relies in general upon strong inclusion systems for $\operatorname{Mod}^{\mathcal{I}}(\Sigma)$ (see Ex. 5.3, Ex. 5.4 and Ex. 5.5) which prohibits the preservation by sub-models of some of the base institution atomic sentences. For example, an atom $\pi$ in $P L$ is not preserved by sub-models of the strong inclusion system of $\operatorname{Mod}^{P L}(P)$ and hence it is also not preserved by sub-models in $\operatorname{Mod}^{\mathcal{H} P L}(P, \mathrm{Nom})$. This means that we cannot make use of Thm. 5.2 in conjunction with Cor. 7.2 in order to establish that sentences such as $@_{i} \pi$ may be used for initial semantics specifications. However, sentences such as $@_{i} \pi$ are quite expected properties to be stated at the level of formal specifications.

Fortunately there is a simple technical solution to this problem. The sentences that somehow fall outside the scope of the application of Thm. 5.2 may sometimes instead be considered as constraints on the models. In our example of $@_{i} \pi$ this means that we consider the sub-functor $\operatorname{Mod}^{\prime}$ of $\operatorname{Mod}^{\mathcal{H} P L}$ determined by the models $(M, R)$ that satisfy $(M, R) \models @_{i} \pi$. It is rather straightforward to check that the conditions of Cor. 7.2 are still satisfied, the most relevant part being in fact the checking of the conditions of Thm. 5.2 for $\operatorname{Mod}^{\prime}(P$, Nom $)$.

\section{Conclusion}

In this paper

- we have recalled the hybridization of institutions developed in [30] and have upgraded it to the abstract concept of constrained models, 
- we have developed a concept of quasi-variety for models of hybridized institutions, the most technical part being that of establishing general conditions for inclusion systems for categories of models of hybridized institutions,

- we have developed preservation results by sub-models and by products in hybridized institutions; in this process we have introduced notions of 'local' preservation, and

- we have put all the above results together for developing a general method and result for the existence of initial semantics in hybridized institutions.

Given the high level of abstraction of our developments, our result are applicable to a wide variety of hybrid logics, that include various logical system at the base level, employ many forms of quantifications, and constrain the models in numerous ways. This is especially important within the current context of the high proliferation in the number of modal and hybrid logics for formal specification, including various logical combinations underlying computing paradigms combinations.

\section{Acknowledgement}

The author thanks the first referee for very careful reading of the work and for providing useful feedback. This work has been supported by a grant of the Romanian National Authority for Scientific Research, CNCS-UEFISCDI, project number PN-II-ID-PCE-2011-3-0439.

\section{References}

[1] Marc Aiguier and Fabrice Barbier. An institution-independent proof of the Beth definability theorem. Studia Logica, 85(3):333-359, 2007.

[2] Marc Aiguier and Răzvan Diaconescu. Stratified institutions and elementary homomorphisms. Information Processing Letters, 103(1):5-13, 2007.

[3] Hajnal Andréka and István Németi. Generalization of the concept of variety and quasivariety to partial algebras through category theory. Dissertationes Mathematicae, CCIV, 1983.

[4] Carlos Areces, Patrick Blackburn, and Samuel R. Delany. Bringing them all together. Journal of Logic and Computation, 11:657-669, 2001.

[5] Edigio Astesiano, Michel Bidoit, Hélène Kirchner, Berndt Krieg-Brückner, Peter Mosses, Don Sannella, and Andrzej Tarlecki. CASL: The common algebraic specification language. Theoretical Computer Science, 286(2):153-196, 2002.

[6] Jean-Yves Béziau. 13 questions about universal logic. Bulletin of the Section of Logic, 35(2/3):133-150, 2006.

[7] Patrick Blackburn. Representation, reasoning, and relational structures: a hybrid logic manifesto. Logic Journal of IGPL, 8, 2000.

[8] Francis Borceux. Handbook of Categorical Algebra. Cambridge University Press, 1994.

[9] Torben Braüner. Natural deduction for first-order hybrid logic. Journal of Logic, Language and Information, 14:173, 2005.

[10] Torben Braüner. Hybrid Logic and its Proof-Theory, volume 37 of Applied Logic Series. Springer, 2011.

[11] Rod Burstall and Joseph Goguen. The semantics of Clear, a specification language. In Dines Bjorner, editor, 1979 Copenhagen Winter School on Abstract Software Specification, volume 86 of Lecture Notes in Computer Science, pages 292-332. Springer, 1980.

[12] Virgil Emil Căzănescu and Grigore Roşu. Weak inclusion systems. Mathematical Structures in Computer Science, 7(2):195206, 1997.

[13] Răzvan Diaconescu. Elementary diagrams in institutions. Journal of Logic and Computation, 14(5):651-674, 2004.

[14] Răzvan Diaconescu. Institution-independent Model Theory. Birkhäuser, 2008.

[15] Răzvan Diaconescu. Quasi-boolean encodings and conditionals in algebraic specification. Journal of Logic and Algebraic Programming, 79(2):174-188, 2010.

[16] Răzvan Diaconescu. Grothendieck inclusion systems. Applied Categorical Structures, 19(5):783-802, 2011.

[17] Răzvan Diaconescu and Ionuţ Ţuţu. On the algebra of structured specifications. Theoretical Computer Science, 412(28):3145-3174, 2011.

[18] Răzvan Diaconescu, Joseph Goguen, and Petros Stefaneas. Logical support for modularisation. In Gerard Huet and Gordon Plotkin, editors, Logical Environments, pages 83-130. Cambridge, 1993. Proceedings of a Workshop held in Edinburgh, Scotland, May 1991. 
[19] Răzvan Diaconescu and Petros Stefaneas. Ultraproducts and possible worlds semantics in institutions. Theoretical Computer Science, 379(1):210-230, 2007.

[20] Joseph Goguen and Rod Burstall. Institutions: Abstract model theory for specification and programming. Journal of the Association for Computing Machinery, 39(1):95-146, 1992.

[21] Joseph Goguen and José Meseguer. Models and equality for logical programming. In Hartmut Ehrig, Giorgio Levi, Robert Kowalski, and Ugo Montanari, editors, Proceedings, TAPSOFT 1987, volume 250 of Lecture Notes in Computer Science, pages 1-22. Springer, 1987.

[22] Joseph Goguen and Grigore Roşu. Composing hidden information modules over inclusive institutions. In From ObjectOrientation to Formal Methods, volume 2635 of Lecture Notes in Computer Science, pages 96-123. Springer, 2004.

[23] Joseph Goguen, James Thatcher, Eric Wagner, and Jesse Wright. Initial algebra semantics and continuous algebras. Journal of the Association for Computing Machinery, 24(1):68-95, January 1977. An early version is "Initial Algebra Semantics", with James Thatcher, IBM T.J. Watson Research Center, Report RC 4865, May 1974.

[24] George Grätzer. Universal Algebra. Springer, 1979.

[25] H. Kaphengst and Horst Reichel. Initial algebraic semantics for non-context-free languages. In Marek Karpinski, editor, Fundamentals of Computation Theory, pages 120-126. Springer, 1977. Lecture Notes in Computer Science, Volume 56.

[26] Saunders Mac Lane. Categories for the Working Mathematician. Springer, second edition, 1998.

[27] John Lloyd. Foundations of Logic Programming. Springer, 1984.

[28] Anatoly Malcev. The Metamathematics of Algebraic Systems. North-Holland, 1971.

[29] Manuel Martins, Alexandre Madeira, and Luis Barbosa. Reasoning about complex requirements in a uniform setting. Electronic Proceeding of TICTTL, Third International Congress in Tools for Teaching Logic, 1-4 June Salamanca, 2011.

[30] Manuel-Antonio Martins, Alexandre Madeira, Răzvan Diaconescu, and Luis Barbosa. Hybridization of institutions. In Andrea Corradini, Bartek Klin, and Corina Cîrstea, editors, Algebra and Coalgebra in Computer Science, volume 6859 of Lecture Notes in Computer Science, pages 283-297. Springer, 2011.

[31] Solomon Passy and Tinko Tinchev. An essay in combinatory dynamic logic. Information and Computation, 93(2):263-332, 1991

[32] Arthur N. Prior. Past, Present and Future. Oxford University Press, 1967.

[33] Grigore Roşu. Axiomatisability in inclusive equational logic. Mathematical Structures in Computer Science, 12(5):541-563, 2002.

[34] Andrzej Tarlecki. Bits and pieces of the theory of institutions. In David Pitt, Samson Abramsky, Axel Poigné, and David Rydeheard, editors, Proceedings, Summer Workshop on Category Theory and Computer Programming, volume 240 of Lecture Notes in Computer Science, pages 334-360. Springer, 1986.

[35] Andrzej Tarlecki. Quasi-varieties in abstract algebraic institutions. Journal of Computer and System Sciences, 33(3):333-360, 1986.

[36] Andrzej Tarlecki, Rod Burstall, and Joseph Goguen. Some fundamental algebraic tools for the semantics of computation, part 3: Indexed categories. Theoretical Computer Science, 91:239-264, 1991. 